TRANSACTIONS OF THE

AMERICAN MATHEMATICAL SOCIETY

Volume 365, Number 12, December 2013, Pages 6403-6440

S 0002-9947(2013)05861-8

Article electronically published on April 18, 2013

\title{
DISPERSIVE ESTIMATES FOR SCHRÖDINGER OPERATORS IN DIMENSION TWO WITH OBSTRUCTIONS AT ZERO ENERGY
}

\author{
M. BURAK ERDOĞAN AND WILLIAM R. GREEN
}

\begin{abstract}
We investigate $L^{1}\left(\mathbb{R}^{2}\right) \rightarrow L^{\infty}\left(\mathbb{R}^{2}\right)$ dispersive estimates for the Schrödinger operator $H=-\Delta+V$ when there are obstructions, resonances or an eigenvalue, at zero energy. In particular, we show that the existence of an $\mathrm{s}$-wave resonance at zero energy does not destroy the $t^{-1}$ decay rate. We also show that if there is a p-wave resonance or an eigenvalue at zero energy, then there is a time dependent operator $F_{t}$ satisfying $\left\|F_{t}\right\|_{L^{1} \rightarrow L^{\infty}} \lesssim 1$ such that

$$
\left\|e^{i t H} P_{a c}-F_{t}\right\|_{L^{1} \rightarrow L^{\infty}} \lesssim|t|^{-1}, \quad \text { for }|t|>1 \text {. }
$$
\end{abstract}

We also establish a weighted dispersive estimate with $t^{-1}$ decay rate in the case when there is an eigenvalue at zero energy but no resonances.

\section{INTRODUCTION}

Consider the Schrödinger operator $H=-\Delta+V$ in $\mathbb{R}^{2}$, where $V$ is a real-valued potential. Let $P_{a c}$ be the orthogonal projection onto the absolutely continuous subspace of $L^{2}\left(\mathbb{R}^{2}\right)$, which is determined by $H$. In [24], Schlag proved that

$$
\left\|e^{i t H} P_{a c}\right\|_{L^{1}\left(\mathbb{R}^{2}\right) \rightarrow L^{\infty}\left(\mathbb{R}^{2}\right)} \lesssim|t|^{-1}
$$

under the decay assumption $|V| \lesssim\langle x\rangle^{-3-}$ and the assumption that zero is neither an eigenvalue nor a resonance of $H$.

Recall that (see, e.g., [15] or Section 5 below) there is a resonance at zero energy if there is a distributional solution to the equation $H \psi=0$ where $\psi \notin L^{2}\left(\mathbb{R}^{2}\right)$ but $\psi \in L^{p}\left(\mathbb{R}^{2}\right)$ for some $p \in(2, \infty]$. There are two possible cases, either $\psi \in L^{\infty}\left(\mathbb{R}^{2}\right)$ and $\psi \notin L^{p}\left(\mathbb{R}^{2}\right)$ for any $p<\infty$ or $\psi \in L^{p}\left(\mathbb{R}^{2}\right)$ for all $p \in(2, \infty]$. In the case of $\psi \in L^{\infty}\left(\mathbb{R}^{2}\right)$ only, the resonance is called an s-wave resonance. In the second case, we say there is a p-wave resonance. We say that there is an eigenvalue at zero if $\psi \in L^{2}\left(\mathbb{R}^{2}\right)$. This definition for resonances differs from the case of dimension $n=3$ in which $\psi$ lies in weighted $L^{2}$ spaces.

We note that in the case of $V \equiv 0$ the function $\psi \equiv 1$ solves $H \psi=0$ which corresponds to an s-wave resonance. It is important to note that in spite of this obstruction, the free evolution decays in time at the rate $t^{-1}$.

Much is known about dispersive estimates for the Schrödinger equation when zero is regular. The history goes back to Rauch, 20], who studied the local decay in dimension three. In [20, he noted that in the generic case, i.e. when there may be eigenvalues or resonances, the evolution decays at a rate of $|t|^{-1 / 2}$ as $t \rightarrow \infty$ on exponentially weighted $L^{2}$ spaces. In the case when there are no eigenvalues

Received by the editors January 11, 2012 and, in revised form, April 19, 2012.

2010 Mathematics Subject Classification. Primary 35J10.

The first author was partially supported by NSF grant DMS-0900865. 
or resonances, it was shown that the decay rate is $|t|^{-3 / 2}$. Jensen and Kato, 14, improved this result to polynomially weighted $L^{2}$ spaces in dimension three and in higher dimensions, [12, 13. In [14, it was noted that the presence of a zero energy eigenvalue or resonance destroys the $|t|^{-3 / 2}$ decay even if one projects away from the eigenspace in dimension three.

Local decay estimates in the two dimensional case were studied by Murata in [19]. In the case when zero is a regular point of the spectrum, Murata was able to prove an estimate on weighted $L^{2}$ spaces that decays like $t^{-1}(\log t)^{-2}$, which is integrable at infinity 1 Such estimates have been used in analysis of the stability of certain two dimensional non-linear equations. It is important to note that the free evolution does not satisfy this estimate. In the general case, the following bound is implicit in [19]:

$$
e^{i t H} P_{a c}(H)=F_{t}+O(1 / t), \quad t>2,
$$

where $F_{t}$ is a time dependent finite rank operator between certain weighted $L^{2}$ spaces with operator norm $\lesssim \frac{1}{\log (t)}$. The error term is also understood in the weighted $L^{2}$ setting.

The first result to discuss global decay, $L^{1} \rightarrow L^{\infty}$ estimates, was due to Journé, Soffer and Sogge in [17]. Their result relied on the integrability of $t^{-n / 2}$ at infinity and is thus restricted to $n \geq 3$. Much is now known in this direction, mainly in dimension three. Rodnianski and Schlag established such estimates in dimension three, [22], in addition to establishing Strichartz estimates. Following their methods, a great number of results in dimension three ensued, particularly [9, 10, 11]. The one dimensional problem was studied by Weder [26], and Goldberg and Schlag [11. Also see [6, 28, 8, for global estimates in the three dimensional case when there is an eigenvalue and/or resonance at zero energy, and [7] for a similar result for the matrix Schrödinger equation.

There have also been studies of the wave-operators in dimension two. In particular Yajima [27], established that the wave operators are bounded on $L^{p}\left(\mathbb{R}^{2}\right)$ for $1<p<\infty$ if zero is regular. The hypotheses on the potential $V$ were relaxed slightly in 16. This result would imply global dispersive estimates if extended to the full range of $p, 1 \leq p \leq \infty$. High frequency dispersive estimates, similar to those obtained in [24] and stated as Theorem 1.3 below, were obtained by Moulin, [18], under an integrability condition on the potential.

In this paper we investigate $L^{1} \rightarrow L^{\infty}$ dispersive estimates in $\mathbb{R}^{2}$ when zero energy is not a regular point of the spectrum of the operator $H=-\Delta+V$. Our goal is to prove the following theorem.

Theorem 1.1. Assume that $|V(x)| \lesssim\langle x\rangle^{-\beta}$. If there is only an s-wave resonance at zero energy, then for $\beta>4$, we have

$$
\left\|e^{i t H} P_{a c}(H)\right\|_{L^{1} \rightarrow L^{\infty}} \lesssim|t|^{-1} .
$$

If there is a p-wave resonance or eigenvalue at zero, then for $\beta>6$, there is a time-dependent operator $F_{t}$ such that

$$
\left\|e^{i t H} P_{a c}(H)-F_{t}\right\|_{L^{1} \rightarrow L^{\infty}} \lesssim|t|^{-1}, \quad|t|>1,
$$

\footnotetext{
${ }^{1}$ During the review process of this paper, the authors extended Murata's result to the weighted $L^{1} \rightarrow L^{\infty}$ setting; see [5].
} 
with

$$
\sup _{t}\left\|F_{t}\right\|_{L^{1} \rightarrow L^{\infty}} \lesssim 1
$$

Note that it is natural to have the $t^{-1}$ decay rate in the case of an s-wave resonance since the free Schrödinger has an s-wave resonance at zero energy. The reason that we cannot get any decay in the case of a p-wave resonance or the zero eigenvalue is the behavior of the resolvent around zero energy. In the three dimensional case the resolvent $\left(H-z^{2}\right)^{-1}$ has an expansion of the form

$$
\left(H-z^{2}\right)^{-1}=-G_{-2} z^{-2}+G_{-1} z^{-1}+O(1), z \rightarrow 0, \Im(z)>0 .
$$

The most singular term $G_{-2} z^{-2}$ gives the Riesz projection to zero energy eigenspace. If one projects away from the zero eigenspace, the worst singularity is $\frac{1}{z}$, which allows for $|t|^{-1 / 2}$ decay as $t \rightarrow \pm \infty$; see [6]. However, in the two dimensional case the resolvent expansion around zero contains logarithmic terms. In particular, in the general case of zero energy resonances (even if one projects away the zero energy eigenspace), the most singular term is of the form $\frac{1}{z^{2} \log (z)}$, which does not allow for any polynomial decay in $t$. In light of (1), it should be possible to prove that $F_{t}$ is of finite rank and that it decays at the rate $\frac{1}{\log (t)}$. We won't pursue these issues in this paper. It turns out that it is possible to improve Theorem 1.1 in the case when zero is an eigenvalue but there are no resonances at zero. In particular, we show the following.

Theorem 1.2. Assume that $|V(x)| \lesssim\langle x\rangle^{-\beta}$ for some $\beta>11$. If zero is an eigenvalue of $H=-\Delta+V$ and there are neither $s$-wave nor $p$-wave resonances at zero, then

$$
\left\|e^{i t H} P_{a c}\right\|_{L^{1,1+} \rightarrow L^{\infty,-1-}} \lesssim|t|^{-1} .
$$

Here $L^{1,1+}$ is the weighted $L^{1}$ space defined by

$$
L^{1,1+}\left(\mathbb{R}^{2}\right):=\left\{f: \int_{\mathbb{R}^{2}}|f(x)|\langle x\rangle^{1+} d x<\infty\right\} .
$$

Similarly, $L^{\infty,-1-}=\left\{f:\langle x\rangle^{-1-} f \in L^{\infty}\right\}$.

Let $\chi$ be an even smooth function supported in $\left[-\lambda_{1}, \lambda_{1}\right]$ and $\chi(x)=1$ for $|x|<\lambda_{1} / 2$. Let $K_{\lambda_{1}}$ be the kernel of $e^{i t H} \chi(H) P_{a c}$ :

$$
K_{\lambda_{1}}(x, y)=\frac{1}{\pi i} \int_{0}^{\infty} e^{i t \lambda^{2}} \lambda \chi(\lambda)\left[R_{V}^{+}\left(\lambda^{2}\right)-R_{V}^{-}\left(\lambda^{2}\right)\right](x, y) d \lambda,
$$

where

$$
R_{V}^{ \pm}\left(\lambda^{2}\right)=R_{V}\left(\lambda^{2} \pm i 0\right)=\left(H-\left(\lambda^{2} \pm i 0\right)\right)^{-1}
$$

is the perturbed resolvent. By the limiting absorption principle, these boundary values are bounded operators on weighted $L^{2}$-spaces; see e.g. [2].

The high energies were studied in 24]:

Theorem $1.3([24])$. Assume that $|V| \lesssim\langle x\rangle^{-2-}$. Then for any $\lambda_{1}>0$,

$$
\left\|e^{i t H} P_{a c}-e^{i t H} \chi(H) P_{a c}\right\|_{1 \rightarrow \infty} \leq C_{\lambda_{1}}|t|^{-1} .
$$

Therefore, in the proof of Theorem 1.1 and Theorem 1.2, it suffices to obtain the stated bounds for the operator $K_{\lambda_{1}}$ for some $\lambda_{1}>0$. Our analysis relies on expansions of the resolvent operator at zero energy following those of [15]; see also 
the previous work in $[3,4$. We repeat part of the argument to obtain more flexible and favorable error bounds for our purposes.

We also note that standard spectral theoretic results for $H$ apply. Under our assumptions we have that the spectrum of $H$ can be expressed as the absolutely continuous spectrum, the interval $[0, \infty)$, and finitely many eigenvalues of finite multiplicity on $(-\infty, 0]$. See [21] for spectral theory and 25] for Birman-Schwinger type bounds.

Our paper is organized as follows. We set out the necessary expansions for the resolvent in Section 2. We then study $K_{\lambda_{1}}$ to establish Theorem 1.1 in the case when there is an s-wave resonance at zero in Section 3. In Section 4 we establish Theorem 1.1 in the case of a p-wave resonance or eigenvalue at zero energy. In Section 5 we discuss the spectral structure of $-\Delta+V$ at zero energy. Finally we prove Theorem 1.2 in Section 6.

\section{RESOlVENT EXPANSIONS AROUND ZERO ENERGY IN THE CASE OF AN S-WAVE RESONANCE}

In this section, following [15], we obtain resolvent expansions around the threshold $\lambda=0$ in the case when there is only s-wave resonance at zero (resonance of the first kind; see Definition 2.3 below and the remarks following it). We now introduce some definitions and notation.

Definition 2.1. We say an operator $T: L^{2}\left(\mathbb{R}^{2}\right) \rightarrow L^{2}\left(\mathbb{R}^{2}\right)$ with kernel $T(\cdot, \cdot)$ is absolutely bounded if the operator with kernel $|T(\cdot, \cdot)|$ is bounded from $L^{2}\left(\mathbb{R}^{2}\right)$ to $L^{2}\left(\mathbb{R}^{2}\right)$.

It is worth noting that a Hilbert-Schmidt operator is an absolutely bounded operator.

We say that an absolutely bounded operator $T(\lambda)(\cdot, \cdot)$ is $\mathcal{O}_{1}\left(\lambda^{s}\right)$ if the integral kernel satisfies the following estimates:

$$
\left\|\sup _{0<\lambda<\lambda_{1}} \lambda^{-s}|T(\lambda)(\cdot, \cdot)|\right\|_{L^{2} \rightarrow L^{2}} \lesssim 1,\left\|\sup _{0<\lambda<\lambda_{1}} \lambda^{1-s} \mid \partial_{\lambda} T(\lambda)(\cdot, \cdot)\right\| \|_{L^{2} \rightarrow L^{2}} \lesssim 1 .
$$

If only the first bound in (3) holds, we say that $T(\lambda)(\cdot, \cdot)$ is $O\left(\lambda^{s}\right)$. We also note that we can replace $\lambda^{-s}$ with $f(\lambda)^{-1}$ in (3), in which case we say $T(\lambda)(\cdot, \cdot)$ is $O(f(\lambda))$.

Recall that

$$
R_{0}^{ \pm}\left(\lambda^{2}\right)(x, y)= \pm \frac{i}{4} H_{0}^{ \pm}(\lambda|x-y|),
$$

where $H_{0}^{ \pm}$are the Hankel functions of order zero:

$$
H_{0}^{ \pm}(z)=J_{0}(z) \pm i Y_{0}(z) .
$$

From the series expansions for the Bessel functions (see [1]), as $z \rightarrow 0$ we have

$$
\begin{aligned}
J_{0}(z) & =1-\frac{1}{4} z^{2}+\frac{1}{64} z^{4}+O\left(z^{6}\right)=1+O\left(z^{2}\right) \\
Y_{0}(z) & =\frac{2}{\pi}(\log (z / 2)+\gamma) J_{0}(z)+\frac{2}{\pi}\left(\frac{1}{4} z^{2}-\frac{3}{128} z^{4}+O\left(z^{6}\right)\right) \\
& =\frac{2}{\pi} \log (z)+O(1) .
\end{aligned}
$$


We also have the following estimates for the derivatives as $z \rightarrow 0$ :

$$
J_{0}^{\prime}(z)=O(z), \quad J_{0}^{\prime \prime}(z)=O(1), \quad Y_{0}^{\prime}(z)=\frac{2}{\pi z}+O(1) .
$$

Further, for $|z|>1$, we have the representation (see, e.g., [1])

$$
H_{0}^{ \pm}(z)=e^{ \pm i z} \omega_{ \pm}(z), \quad\left|\omega_{ \pm}^{(\ell)}(z)\right| \lesssim(1+|z|)^{-\frac{1}{2}-\ell}, \quad \ell=0,1,2, \ldots
$$

This implies that for $|z|>1$,

(10) $\mathcal{C}(z)=e^{i z} \omega_{+}(z)+e^{-i z} \omega_{-}(z)$,

$$
\left|\omega_{ \pm}^{(\ell)}(z)\right| \lesssim(1+|z|)^{-\frac{1}{2}-\ell}, \quad \ell=0,1,2, \ldots,
$$

for any $\mathcal{C} \in\left\{J_{0}, Y_{0}\right\}$ respectively with different $\omega_{ \pm}$.

Let $U(x)=1$ if $V(x) \geq 0$ and $U(x)=-1$ if $V(x)<0$, and let $v=|V|^{1 / 2}$. We have $V=U v^{2}$. We use the symmetric resolvent identity, valid for $\Im \lambda>0$ :

$$
R_{V}^{ \pm}\left(\lambda^{2}\right)=R_{0}^{ \pm}\left(\lambda^{2}\right)-R_{0}^{ \pm}\left(\lambda^{2}\right) v M^{ \pm}(\lambda)^{-1} v R_{0}^{ \pm}\left(\lambda^{2}\right)
$$

where $M^{ \pm}(\lambda)=U+v R_{0}^{ \pm}\left(\lambda^{2}\right) v$. The key issue in the resolvent expansions is the invertibility of the operator $M^{ \pm}(\lambda)$ for small $\lambda$ under various spectral assumptions at zero. Below, we obtain expansions of the operator $M^{ \pm}(\lambda)$ around $\lambda=0$ using the properties of the free resolvent listed above. A similar lemma was proved in [24]; however, we need to expand the operator further and obtain slightly more general error bounds. The following operators arise naturally in the expansion of $M^{ \pm}(\lambda)($ see (5),$(60)$ ):

$$
\begin{aligned}
& G_{0} f(x)=-\frac{1}{2 \pi} \int_{\mathbb{R}^{2}} \log |x-y| f(y) d y, \\
& G_{1} f(x)=\int_{\mathbb{R}^{2}}|x-y|^{2} f(y) d y, \\
& G_{2} f(x)=\frac{1}{8 \pi} \int_{\mathbb{R}^{2}}|x-y|^{2} \log |x-y| f(x) d y .
\end{aligned}
$$

Lemma 2.2. For $\lambda>0$ define $M^{ \pm}(\lambda):=U+v R_{0}^{ \pm}\left(\lambda^{2}\right) v$. Let $P=v\langle\cdot, v\rangle\|V\|_{1}^{-1}$ denote the orthogonal projection onto $v$. Then

$$
M^{ \pm}(\lambda)=g^{ \pm}(\lambda) P+T+M_{0}^{ \pm}(\lambda) .
$$

Here $g^{ \pm}(\lambda)=a \ln \lambda+z$, where $a \in \mathbb{R} \backslash\{0\}$ and $z \in \mathbb{C} \backslash \mathbb{R}$, and $T=U+v G_{0} v$, where $G_{0}$ is an integral operator defined in (12). Further, for any $\frac{1}{2} \leq k<2$,

$$
M_{0}^{ \pm}(\lambda)=\mathcal{O}_{1}\left(\lambda^{k}\right)
$$

if $v(x) \lesssim\langle x\rangle^{-\beta}$ for some $\beta>1+k$. Moreover,

$$
M_{0}^{ \pm}(\lambda)=g_{1}^{ \pm}(\lambda) v G_{1} v+\lambda^{2} v G_{2} v+M_{1}^{ \pm}(\lambda) .
$$

Here $G_{1}, G_{2}$ are integral operators defined in (13), (14), and $g_{1}^{ \pm}(\lambda)=\lambda^{2}(\alpha \log \lambda+$ $\left.\beta_{ \pm}\right)$, where $\alpha \in \mathbb{R} \backslash\{0\}$ and $\beta_{ \pm} \in \mathbb{C} \backslash \mathbb{R}$. Further, for any $2<\ell<4$,

$$
M_{1}^{ \pm}(\lambda)=\mathcal{O}_{1}\left(\lambda^{\ell}\right)
$$

if $\beta>1+\ell$.

Proof. The first part with $k=\frac{1}{2}$ was proven in [24, Lemma 5]. To obtain the expansions recall that, for $\lambda>0$,

$$
R_{0}^{ \pm}\left(\lambda^{2}\right)(x, y)= \pm \frac{i}{4} H_{0}^{ \pm}(\lambda|x-y|) .
$$


Using the definition of $H_{0}^{ \pm}(z)$ and the expansions (5) and (6) around $z=0$, we have

$$
\begin{aligned}
\pm \frac{i}{4} H_{0}^{ \pm}(z) & = \pm \frac{i}{4} J_{0}(z)-\frac{1}{4} Y_{0}(z) \\
& =-\frac{1}{2 \pi} \log (z / 2) \pm \frac{i}{4}-\frac{\gamma}{2 \pi}+\alpha z^{2} \log z+\beta_{ \pm} z^{2}+O\left(z^{4} \log z\right) \\
& =-\frac{1}{2 \pi} \log (z / 2) \pm \frac{i}{4}-\frac{\gamma}{2 \pi}+O\left(z^{2} \log z\right)
\end{aligned}
$$

with $\alpha=1 / 8 \pi$ and $\beta_{ \pm} \in \mathbb{C}$. The expansions are now obtained by setting $z=$ $\lambda|x-y|$. In particular, we see

$$
g^{ \pm}(\lambda)=-\|V\|_{1}\left(\frac{1}{2 \pi} \log (\lambda / 2)+\frac{1}{2 \pi} \gamma \mp \frac{i}{4}\right) .
$$

Noting that

$$
\begin{aligned}
& M_{0}^{ \pm}(\lambda)=\left[U+v R_{0}^{ \pm}\left(\lambda^{2}\right) v\right]-\left[g^{ \pm}(\lambda) P+U+v G_{0} v\right], \\
& M_{1}^{ \pm}(\lambda)=\left[U+v R_{0}^{ \pm}\left(\lambda^{2}\right) v\right]-\left[g^{ \pm}(\lambda) P+U+v G_{0} v+g_{1}^{ \pm}(\lambda) v G_{1} v+\lambda^{2} v G_{2} v\right] .
\end{aligned}
$$

Using (17) and (16) for $M_{0}$ and $M_{1}$ respectively, we obtain for $z=\lambda|x-y|<1$ that

$$
\begin{aligned}
\left|M_{0}^{ \pm}(\lambda)(x, y) \chi_{\{\lambda|x-y|<1\}}\right| & \lesssim v(x) v(y)(\lambda|x-y|)^{2} \log (\lambda|x-y|) \\
& \lesssim v(x) v(y)(\lambda|x-y|)^{2-}, \\
\left|M_{1}^{ \pm}(\lambda)(x, y) \chi_{\{\lambda|x-y|<1\}}\right| & \lesssim v(x) v(y)(\lambda|x-y|)^{4} \log (\lambda|x-y|) \\
& \lesssim v(x) v(y)(\lambda|x-y|)^{4-} .
\end{aligned}
$$

For large $z$, using the expansion of the Hankel function about $z=\infty$ (recall (91)), we have $\left|H_{0}^{ \pm}(z)\right| \lesssim 1$ and $\left|\frac{d}{d z} H_{0}^{ \pm}(z)\right| \lesssim z^{-1 / 2}$. Thus for large $z>1$, for $M_{0}^{ \pm}(z)$ the $\log z$ term dominates and for $M_{1}^{ \pm}(z)$ the $z^{2} \log z$ term in (16) dominates, and we have

$$
\begin{aligned}
\left|M_{0}^{ \pm}(\lambda)(x, y) \chi_{\{\lambda|x-y|>1\}}\right| & \lesssim v(x) v(y) \log (\lambda|x-y|) \chi_{\{\lambda|x-y|>1\}} \\
& \lesssim v(x) v(y)(\lambda|x-y|)^{0+} \chi_{\{\lambda|x-y|>1\}}, \\
\left|M_{1}^{ \pm}(\lambda)(x, y) \chi_{\{\lambda|x-y|>1\}}\right| & \lesssim v(x) v(y)(\lambda|x-y|)^{2+} \chi_{\{\lambda|x-y|>1\}} .
\end{aligned}
$$

Hence, for any $0<k<2$ and for any $2<\ell<4$ we have

$$
\begin{aligned}
\left|M_{0}^{ \pm}(\lambda)(x, y)\right| & \lesssim v(x) v(y)\left[(\lambda|x-y|)^{2-} \chi_{\{\lambda|x-y|<1\}}+(\lambda|x-y|)^{0+} \chi_{\{\lambda|x-y|>1\}}\right] \\
& \lesssim v(x) v(y)(\lambda|x-y|)^{k}, \\
\left|M_{1}^{ \pm}(\lambda)(x, y)\right| & \lesssim v(x) v(y)\left[(\lambda|x-y|)^{4-} \chi_{\{\lambda|x-y|<1\}}+(\lambda|x-y|)^{2+} \chi_{\{\lambda|x-y|>1\}}\right] \\
& \lesssim v(x) v(y)(\lambda|x-y|)^{\ell} .
\end{aligned}
$$

This yields the claim for $M_{0}$ and $M_{1}$ since $v(x) v(y)|x-y|^{\ell}$ is Hilbert-Schmidt from $L^{2}\left(\mathbb{R}^{2}\right)$ to $L^{2}\left(\mathbb{R}^{2}\right)$ for $\beta>1+\ell$. For $\lambda$-derivatives, we note that

$$
\left|\partial_{\lambda} R_{0}^{ \pm}\left(\lambda^{2}\right)(x, y)\right| \lesssim\left(\frac{|x-y|}{\lambda}\right)^{\frac{1}{2}}
$$


and

$$
\partial_{\lambda} F(\lambda|x-y|)=\left.|x-y| \partial_{z} F(z)\right|_{z=\lambda|x-y|} .
$$

For the terms in $M_{0}$ and $M_{1}$ other than $R_{0}$, the effect of $\partial_{\lambda}$ is comparable to division by $\lambda$. However, due to (19), on $\lambda|x-y|>1$ we have for any $k \geq \frac{1}{2}$,

$$
\left|\partial_{\lambda} M_{0}(\lambda)(x, y)\right| \lesssim v(x) v(y)\left[\left(\frac{|x-y|}{\lambda}\right)^{\frac{1}{2}}+\lambda^{-1}\right] \lesssim v(x) v(y) \lambda^{k-1}|x-y|^{k} .
$$

Similarly,

$$
\begin{aligned}
\left|\partial_{\lambda} M_{1}(\lambda)(x, y)\right| & \lesssim v(x) v(y)\left[\left(\frac{|x-y|}{\lambda}\right)^{\frac{1}{2}}+\lambda^{-1}(\lambda|x-y|)^{\ell}\right] \\
& \lesssim v(x) v(y) \lambda^{\ell-1}|x-y|^{\ell} .
\end{aligned}
$$

We now give the definition of resonances from [15]; see also [24. Recall that $Q:=\mathbb{1}-P$.

Definition 2.3. (1) We say zero is a regular point of the spectrum of $H=$ $-\Delta+V$ provided $Q T Q=Q\left(U+v G_{0} v\right) Q$ is invertible on $Q L^{2}\left(\mathbb{R}^{2}\right)$.

(2) Assume that zero is not a regular point of the spectrum. Let $S_{1}$ be the Riesz projection onto the kernel of $Q T Q$ as an operator on $Q L^{2}\left(\mathbb{R}^{2}\right)$. Then $Q T Q+S_{1}$ is invertible on $Q L^{2}\left(\mathbb{R}^{2}\right)$. Accordingly, we define $D_{0}=(Q T Q+$ $\left.S_{1}\right)^{-1}$ as an operator on $Q L^{2}\left(\mathbb{R}^{2}\right)$. We say there is a resonance of the first kind at zero if the operator $T_{1}:=S_{1} T P T S_{1}$ is invertible on $S_{1} L^{2}\left(\mathbb{R}^{2}\right)$.

(3) We say there is a resonance of the second kind at zero if $T_{1}$ is not invertible on $S_{1} L^{2}\left(\mathbb{R}^{2}\right)$ but $T_{2}:=S_{2} v G_{1} v S_{2}$ is invertible on $S_{2} L^{2}\left(\mathbb{R}^{2}\right)$, where $S_{2}$ is the Riesz projection onto the kernel of $T_{1}$ (recall the definition of $G_{1}$ and $G_{2}$ in (13) and (14)).

(4) Finally, if $T_{2}$ is not invertible on $S_{2} L^{2}\left(\mathbb{R}^{2}\right)$, we say there is a resonance of the third kind at zero. We note that in this case the operator $T_{3}:=S_{3} v G_{2} v S_{3}$ is always invertible on $S_{3} L^{2}$, where $S_{3}$ is the Riesz projection onto the kernel of $T_{2}$ (see (6.41) in [15] or Section 5 below).

Remarks. i) In [15], it is noted that the projections $S_{1}-S_{2}, S_{2}-S_{3}$ and $S_{3}$ correspond to s-wave resonances, p-wave resonances, and zero eigenspace, respectively. In particular, resonance of the first kind means that there is only an s-wave resonance at zero. Resonance of the second kind means that there is a p-wave resonance and there may or may not be an s-wave resonance. Finally, resonance of the third kind means that zero is an eigenvalue and there may or may not be s-wave and p-wave resonances. We characterize these projections in Section 5 .

ii) Since $Q T Q$ is self-adjoint, $S_{1}$ is the orthogonal projection onto the kernel of $Q T Q$, and we have (with $\left.D_{0}=\left(Q T Q+S_{1}\right)^{-1}\right)$

$$
S_{1} D_{0}=D_{0} S_{1}=S_{1} \text {. }
$$

This statement is also valid for $S_{2}$ and $\left(T_{1}+S_{2}\right)^{-1}$ and for $S_{3}$ and $\left(T_{2}+S_{3}\right)^{-1}$.

iii) The operator $Q D_{0} Q$ is absolutely bounded in $L^{2}$. This was proved in Lemma 8 of [24] in the case $S_{1}=0$. With minor modifications, the same proof works in our case, too.

iv) The operators with kernel $v G_{i} v$ are Hilbert-Schmidt operators on $L^{2}\left(\mathbb{R}^{2}\right)$ if $v(x) \lesssim\langle x\rangle^{-\beta}$ for $\beta>\frac{3}{2}$ if $i=1$ and for $\beta>3$ if $i=2,3$. 
To invert $M^{ \pm}(\lambda)=U+v R_{0}^{ \pm}\left(\lambda^{2}\right) v$, for small $\lambda$, we will use the following lemma (see Lemma 2.1 in [15]) repeatedly.

Lemma 2.4. Let $A$ be a closed operator on a Hilbert space $\mathcal{H}$ and $S$ be a projection. Suppose $A+S$ has a bounded inverse. Then $A$ has a bounded inverse if and only if

$$
B:=S-S(A+S)^{-1} S
$$

has a bounded inverse in $S \mathcal{H}$, and in this case

$$
A^{-1}=(A+S)^{-1}+(A+S)^{-1} S B^{-1} S(A+S)^{-1} .
$$

We will apply this lemma with $A=M^{ \pm}(\lambda)$ and $S=S_{1}$. Thus, we need to show that $M^{ \pm}(\lambda)+S_{1}$ has a bounded inverse in $L^{2}\left(\mathbb{R}^{2}\right)$ and

$$
B_{ \pm}=S_{1}-S_{1}\left(M^{ \pm}(\lambda)+S_{1}\right)^{-1} S_{1}
$$

has a bounded inverse in $S_{1} L^{2}\left(\mathbb{R}^{2}\right)$. We prove these claims and obtain expansions for the inverses for each type of resonance in Lemma 2.5, Proposition [2.6, and Proposition 4.1 below.

Lemma 2.5. Suppose that zero is not a regular point of the spectrum of $H=$ $-\Delta+V$, and let $S_{1}$ be the corresponding Riesz projection. Then for sufficiently small $\lambda_{1}>0$, the operators $M^{ \pm}(\lambda)+S_{1}$ are invertible for all $0<\lambda<\lambda_{1}$ as bounded operators on $L^{2}\left(\mathbb{R}^{2}\right)$. Further, one has

$$
=h_{ \pm}(\lambda)^{-1} S+Q D_{0} Q+\mathcal{O}_{1}\left(\lambda^{k}\right),
$$

for any $\frac{1}{2} \leq k<2$ if $v(x) \lesssim\langle x\rangle^{-(1+k)-}$. Here $h_{+}(\lambda)=\overline{h_{-}(\lambda)}=a \ln \lambda+z$ (with $a \in \mathbb{R} \backslash\{0\}$ and $z \in \mathbb{C}, \Im z \neq 0)$, and

$$
S=\left[\begin{array}{cc}
P & -P T Q D_{0} Q \\
-Q D_{0} Q T P & Q D_{0} Q T P T Q D_{0} Q
\end{array}\right]
$$

is a finite-rank operator with real-valued kernel.

Proof. We will give the proof for $M^{+}$and drop the superscript "+" from the formulas. Using Lemma 2.2, we write $M(\lambda)+S_{1}$ with respect to the decomposition $L^{2}\left(\mathbb{R}^{2}\right)=P L^{2}\left(\mathbb{R}^{2}\right) \oplus Q L^{2}\left(\mathbb{R}^{2}\right)$.

$$
M(\lambda)+S_{1}=\left[\begin{array}{cc}
g(\lambda) P+P\left(T+S_{1}\right) P & P\left(T+S_{1}\right) Q \\
Q\left(T+S_{1}\right) P & Q\left(T+S_{1}\right) Q
\end{array}\right]+M_{0}(\lambda) .
$$

Noting that $Q \geq S_{1}$, we have $S_{1} P=P S_{1}=0$. Therefore,

$$
M(\lambda)+S_{1}=\left[\begin{array}{cc}
g(\lambda) P+P T P & P T Q \\
Q T P & Q\left(T+S_{1}\right) Q
\end{array}\right]+M_{0}(\lambda) .
$$

Denote the matrix component of the above equation by $A(\lambda)=\left\{a_{i j}(\lambda)\right\}_{i, j=1}^{2}$.

Since $Q\left(T+S_{1}\right) Q$ is invertible, by the Fehsbach formula the invertibility of $A(\lambda)$ hinges upon the existence of $d=\left(a_{11}-a_{12} a_{22}^{-1} a_{21}\right)^{-1}$. Denoting $D_{0}=(Q(T+$ $\left.\left.S_{1}\right) Q\right)^{-1}: Q L^{2} \rightarrow Q L^{2}$, we have

$$
d=\left(g(\lambda) P+P T P-P T Q D_{0} Q T P\right)^{-1}=h(\lambda)^{-1} P
$$

with $h(\lambda)=g(\lambda)+\operatorname{Tr}\left(P T P-P T Q D_{0} Q T P\right)=a \ln (\lambda)+z$, with $a \in \mathbb{R}$ and $z \in \mathbb{C}$. This follows from (18) and the fact that $\operatorname{Tr}\left(P T P-P T Q D_{0} Q T P\right)$ is $\lambda$ independent and real-valued, as the kernels of $T, Q D_{0} Q$ and $v$ are real-valued. Therefore, $d$ exists if $\lambda$ is sufficiently small. 
Thus, by the Fehsbach formula,

$$
\begin{aligned}
A(\lambda)^{-1} & =\left[\begin{array}{cc}
d & -d a_{12} a_{22}^{-1} \\
-a_{22}^{-1} a_{21} d & a_{22}^{-1} a_{21} d a_{12} a_{22}^{-1}+a_{22}^{-1}
\end{array}\right] \\
& =h^{-1}(\lambda)\left[\begin{array}{cc}
P & -P T Q D_{0} Q \\
-Q D_{0} Q T P & Q D_{0} Q T P T Q D_{0} Q
\end{array}\right]+Q D_{0} Q \\
& =: h^{-1}(\lambda) S+Q D_{0} Q .
\end{aligned}
$$

Note that $S$ has rank at most two. This and the absolute boundedness of $Q D_{0} Q$ imply that $A^{-1}(\lambda)=\mathcal{O}_{1}(1)$.

Finally, we write

$$
M(\lambda)+S_{1}=A(\lambda)+M_{0}(\lambda)=\left[\mathbb{1}+M_{0}(\lambda) A^{-1}(\lambda)\right] A(\lambda) .
$$

Since $A^{-1}(\lambda)=\mathcal{O}_{1}(1)$ and, by Lemma 2.2, $M_{0}(\lambda)=\mathcal{O}_{1}\left(\lambda^{k}\right)$ provided $|v(x)| \lesssim$ $\langle x\rangle^{-(1+k)-}$, we obtain

$$
\begin{aligned}
\left(M^{ \pm}(\lambda)+S_{1}\right)^{-1} & =A_{ \pm}^{-1}(\lambda)\left[\mathbb{1}+M_{0}^{ \pm}(\lambda) A_{ \pm}^{-1}(\lambda)\right]^{-1} \\
& =h_{ \pm}(\lambda)^{-1} S+Q D_{0} Q+\mathcal{O}_{1}\left(\lambda^{k}\right)
\end{aligned}
$$

by a Neumann series expansion.

We now prove the invertibility of the operators $B_{ \pm}=S_{1}-S_{1}\left(M^{ \pm}(\lambda)+S_{1}\right)^{-1} S_{1}$.

Proposition 2.6. Assume that $|v(x)| \lesssim\langle x\rangle^{-1-k-}$ for some $k \in\left[\frac{1}{2}, 2\right)$. Then, in the case of a resonance of the first kind, $B_{ \pm}$is invertible on $S_{1} L^{2}\left(\mathbb{R}^{2}\right)$ and we have

$$
B_{ \pm}^{-1}=-h_{ \pm}(\lambda) D_{1}+\mathcal{O}_{1}\left(\lambda^{k}\right),
$$

where $D_{1}=T_{1}^{-1}=\left(S_{1} \text { TPT } S_{1}\right)^{-1}$, and $h_{ \pm}(\lambda)$ is as in Lemma 2.5.

Proof. We again prove the case of the "+" superscripts and subscripts and omit them from the notation. Using Lemma 2.5, we obtain

$$
B=S_{1}-S_{1}\left(h(\lambda)^{-1} S+Q D_{0} Q\right) S_{1}+\mathcal{O}_{1}\left(\lambda^{k+}\right) .
$$

Recall that $S_{1} D_{0}=D_{0} S_{1}=S_{1}$. Further, from the definition (22) of $S$ and the fact that $S_{1} P=P S_{1}=0$, we obtain $S_{1} S S_{1}=S_{1} T P T S_{1}=T_{1}$. Therefore

$$
B=-h(\lambda)^{-1} S_{1} S S_{1}+\mathcal{O}_{1}\left(\lambda^{k+}\right)=-h(\lambda)^{-1} T_{1}+\mathcal{O}_{1}\left(\lambda^{k+}\right) .
$$

Recall that by the definition of a resonance of the first kind, the leading term $T_{1}$ in the definition of $B$ is invertible on $S_{1} L^{2}\left(\mathbb{R}^{2}\right)$. Therefore, for sufficiently small $\lambda$,

$$
\begin{aligned}
B^{-1} & =-h(\lambda)\left[T_{1}-h(\lambda) \mathcal{O}_{1}\left(\lambda^{k+}\right)\right]^{-1}=-h(\lambda)\left[T_{1}+\mathcal{O}_{1}\left(\lambda^{k+}\right)\right]^{-1} \\
& =-h(\lambda) D_{1}+\mathcal{O}_{1}\left(\lambda^{k}\right) .
\end{aligned}
$$

Combining Lemma 2.4, Lemma 2.5, and Proposition 2.6, we obtain

Corollary 2.7. Assume that $|v(x)| \lesssim\langle x\rangle^{-1-k-}$ for some $k \in\left[\frac{1}{2}, 2\right)$. Then in the case of a resonance of the first kind, we have

$$
\begin{aligned}
& M^{ \pm}(\lambda)^{-1}=-h_{ \pm}(\lambda) S_{1} D_{1} S_{1}-S S_{1} D_{1} S_{1}-S_{1} D_{1} S_{1} S \\
& \quad-h_{ \pm}(\lambda)^{-1} S S_{1} D_{1} S_{1} S+h_{ \pm}(\lambda)^{-1} S+Q D_{0} Q+\mathcal{O}_{1}\left(\lambda^{k}\right)
\end{aligned}
$$

provided that $\lambda$ is sufficiently small. 
Proof. Combining Lemma 2.4, Lemma 2.5, and Proposition 2.6, we have

$$
\begin{gathered}
M^{ \pm}(\lambda)^{-1}=\left(M^{ \pm}(\lambda)+S_{1}\right)^{-1}+\left(M^{ \pm}(\lambda)+S_{1}\right)^{-1} S_{1} B^{-1} S_{1}\left(M^{ \pm}(\lambda)+S_{1}\right)^{-1} \\
=h_{ \pm}(\lambda)^{-1} S+Q D_{0} Q-h(\lambda)\left(h_{ \pm}(\lambda)^{-1} S+Q D_{0} Q\right) \\
\times S_{1} D_{1} S_{1}\left(h_{ \pm}(\lambda)^{-1} S+Q D_{0} Q\right)+\mathcal{O}_{1}\left(\lambda^{k}\right) \\
=-h_{ \pm}(\lambda) S_{1} D_{1} S_{1}-S S_{1} D_{1} S_{1}-S_{1} D_{1} S_{1} S-h_{ \pm}(\lambda)^{-1} S S_{1} D_{1} S_{1} S \\
+h_{ \pm}(\lambda)^{-1} S+Q D_{0} Q+\mathcal{O}_{1}\left(\lambda^{k}\right)
\end{gathered}
$$

Here we used the fact that $S_{1} Q D_{0} Q=Q D_{0} Q S_{1}=S_{1}$.

Remark. Under the conditions of Corollary 2.7, the resolvent identity

$$
R_{V}^{ \pm}\left(\lambda^{2}\right)=R_{0}^{ \pm}\left(\lambda^{2}\right)-R_{0}^{ \pm}\left(\lambda^{2}\right) v M^{ \pm}(\lambda)^{-1} v R_{0}^{ \pm}\left(\lambda^{2}\right)
$$

holds as an operator identity on $L^{2, \frac{1}{2}+}\left(\mathbb{R}^{2}\right) \rightarrow L^{2,-\frac{1}{2}-}\left(\mathbb{R}^{2}\right)$, as in the limiting absorption principle, [2].

\section{RESONANCE OF THE FIRST KIND}

In this section, we establish the estimates needed to prove Theorem 1.1. We assume that there is a resonance of the first kind, $\lambda_{1}$ is sufficiently small (so that the analysis in the previous section is valid), and that $v(x) \lesssim\langle x\rangle^{-(1+k)}-$ for $k=1$, or equivalently $|V(x)| \lesssim\langle x\rangle^{-4-}$. It suffices to prove that

Theorem 3.1. Under the conditions above, we have

$$
\left|\left\langle K_{\lambda_{1}} f, g\right\rangle\right| \lesssim|t|^{-1},
$$

for Schwartz functions $f$ and $g$ with $\|f\|_{1}=\|g\|_{1}=1$.

This theorem will be established in Propositions 3.2, 3.11, 3.12, and 3.13, All statements in this section are valid under the conditions above.

Proposition 3.2. The contribution of the first term in Corollary 2.7 to (2) satisfies (28). More explicitly, we have the bound

$$
\begin{aligned}
\mid \int_{\mathbb{R}^{8}} & \int_{0}^{\infty} e^{i t \lambda^{2}} \lambda \chi(\lambda) \mathcal{K}(\lambda, p, q) v\left(x_{1}\right) S_{1} D_{1} S_{1}\left(x_{1}, y_{1}\right) v\left(y_{1}\right) f(x) g(y) d \lambda d x_{1} d y_{1} d x d y \mid \\
& \lesssim|t|^{-1}
\end{aligned}
$$

where $p=\left|x-x_{1}\right|, q=\left|y-y_{1}\right|$, and

$$
\begin{aligned}
& (29) \mathcal{K}(\lambda, p, q)=h^{+}(\lambda) H_{0}^{+}(\lambda p) H_{0}^{+}(\lambda q)-h^{-}(\lambda) H_{0}^{-}(\lambda p) H_{0}^{-}(\lambda q) \\
& =2 i a \log (\lambda)\left[Y_{0}(\lambda p) J_{0}(\lambda q)+J_{0}(\lambda p) Y_{0}(\lambda q)\right]+2 z\left[J_{0}(\lambda p) J_{0}(\lambda q)+Y_{0}(\lambda p) Y_{0}(\lambda q)\right] .
\end{aligned}
$$

To prove this proposition, we need to consider the high and low energy contributions of the Bessel functions separately. To this end we use the partitions of unity $1=\chi\left(\lambda\left|y-y_{1}\right|\right)+\tilde{\chi}\left(\lambda\left|y-y_{1}\right|\right)$ and $1=\chi\left(\lambda\left|x-x_{1}\right|\right)+\tilde{\chi}\left(\lambda\left|x-x_{1}\right|\right)$. We divide the proof of Proposition 3.2 into Lemmas 3.4, 3.8, 3.10 and their respective corollaries, Corollaries 3.5 and 3.9. due to the various terms arising in (29). 
For the low energy parts, the following lemma will be useful:

Lemma 3.3. Let $p=\left|x-x_{1}\right|, q=|x|+1$, and

$$
\begin{aligned}
& F\left(\lambda, x, x_{1}\right):=\chi(\lambda p) Y_{0}(\lambda p)-\chi(\lambda q) Y_{0}(\lambda q), \\
& G\left(\lambda, x, x_{1}\right):=\chi(\lambda p) J_{0}(\lambda p)-\chi(\lambda q) J_{0}(\lambda q) .
\end{aligned}
$$

Then for any $\tau \in[0,1]$ and $\lambda \leq 2 \lambda_{1}$ we have

$$
\begin{aligned}
\left|G\left(\lambda, x, x_{1}\right)\right| & \lesssim \lambda^{\tau}\left\langle x_{1}\right\rangle^{\tau}, \quad\left|\partial_{\lambda} G\left(\lambda, x, x_{1}\right)\right| \lesssim\left\langle x_{1}\right\rangle^{\tau} \lambda^{\tau-1}, \\
\left|F\left(\lambda, x, x_{1}\right)\right| & \leq \int_{0}^{2 \lambda_{1}}\left|\partial_{\lambda} F\left(\lambda, x, x_{1}\right)\right| d \lambda+\left|F\left(0+, x, x_{1}\right)\right| \lesssim k\left(x, x_{1}\right), \\
\left|\partial_{\lambda} F\left(\lambda, x, x_{1}\right)\right| & \lesssim \frac{1}{\lambda} .
\end{aligned}
$$

Here $k\left(x, x_{1}\right):=1+\log ^{+}\left|x_{1}\right|+\log ^{-}\left|x-x_{1}\right|$, where $\log ^{-} y:=\chi_{\{0<y<1\}}|\log y|$ and $\log ^{+} y:=\chi_{\{y>1\}} \log y$.

Proof. We start with $G$. Let $g(s):=\chi(s) J_{0}(s)$. We have $g^{\prime}(s)=O(1)$. Therefore, by the mean value theorem and the boundedness of $g$, we have

$$
\left|G\left(\lambda, x, x_{1}\right)\right| \lesssim \min (\lambda|p-q|, 1) \lesssim \min \left(\lambda\left\langle x_{1}\right\rangle, 1\right) \lesssim \lambda^{\tau}\left\langle x_{1}\right\rangle^{\tau}
$$

for any $0 \leq \tau \leq 1$.

Now consider

$$
\left|\partial_{\lambda} G\left(\lambda, x, x_{1}\right)\right|=\left|p g^{\prime}(\lambda p)-q g^{\prime}(\lambda q)\right| \text {. }
$$

Let $g_{1}(s)=s g^{\prime}(s)$. We have $\left|g_{1}(s)\right| \lesssim 1$ and $\left|g_{1}^{\prime}(s)\right| \lesssim 1$. Therefore, by the mean value theorem and the boundedness of $g_{1}$, we have

$$
\left|\partial_{\lambda} G\left(\lambda, x, x_{1}\right)\right|=\left|\frac{g_{1}(\lambda p)-g_{1}(\lambda q)}{\lambda}\right| \lesssim\left|\frac{\min (\lambda|p-q|, 1)}{\lambda}\right| \lesssim\left\langle x_{1}\right\rangle^{\tau} \lambda^{\tau-1},
$$

for any $\tau \in[0,1]$.

The bounds for $F$ were obtained in 24 . We repeat them for completeness. Note that $F\left(0+, x, x_{1}\right)=\log \left(\frac{\left|x-x_{1}\right|}{|x|+1}\right)+c \lesssim k\left(x, x_{1}\right)$. Therefore it suffices to bound

$$
\begin{aligned}
& \int_{0}^{2 \lambda_{1}}\left|\partial_{\lambda} F\left(\lambda, x, x_{1}\right)\right| d \lambda \lesssim \int_{0}^{2 \lambda_{1}} p\left|\chi^{\prime}(\lambda p) \log (\lambda p)\right| d \lambda \\
& +\int_{0}^{2 \lambda_{1}} q\left|\chi^{\prime}(\lambda q) \log (\lambda q)\right| d \lambda+\int_{0}^{2 \lambda_{1}} \frac{1}{\lambda}|\chi(\lambda p)-\chi(\lambda q)| d \lambda .
\end{aligned}
$$

By inspecting the integrands on the right hand side, we see that $\left|\partial_{\lambda} F\right|$ is bounded by $1 / \lambda$. To obtain the statement for $|F|$ first note that, since $\chi^{\prime}$ is supported in the set $\left[\lambda_{1} / 2,2 \lambda_{1}\right]$, the first line in (30) is $\lesssim 1$. To estimate the second line note that $\chi(\lambda p)-\chi(\lambda q)$ is supported on the set $\left[\frac{\lambda_{1}}{2 p}, 2 \frac{\lambda_{1}}{q}\right]$, which implies that the last line is $\lesssim\left|\log \left(\frac{\left|x-x_{1}\right|}{|x|+1}\right)\right| \lesssim k\left(x, x_{1}\right)$.

Lemma 3.4. We have the bound

$$
\begin{gathered}
\mid \int_{\mathbb{R}^{8}} \int_{0}^{\infty} e^{i t \lambda^{2}} \lambda \chi(\lambda) \log \lambda \chi\left(\lambda\left|x-x_{1}\right|\right) Y_{0}\left(\lambda\left|x-x_{1}\right|\right) v\left(x_{1}\right) S_{1} D_{1} S_{1}\left(x_{1}, y_{1}\right) v\left(y_{1}\right) \\
\left.J_{0}\left(\lambda\left|y-y_{1}\right|\right) \chi\left(\lambda\left|y-y_{1}\right|\right) d \lambda f(x) g(y) d x_{1} d y_{1} d x d y|\lesssim| t\right|^{-1}
\end{gathered}
$$


Proof. Since $S_{1} \leq Q$ are projections and $Q$ is the projection orthogonal to $v$, we have

$$
\int_{\mathbb{R}^{4}} v(x)\left[S_{1} D_{1} S_{1}\right](x, y) h(y) d x d y=\int_{\mathbb{R}^{4}} h(x)\left[S_{1} D_{1} S_{1}\right](x, y) v(y) d x d y=0
$$

for all $h \in L^{2}\left(\mathbb{R}^{2}\right)$. As such, we can subtract functions of $x$ (resp. $y$ ) only from $\chi Y_{0}$ (resp. $\left.\chi J_{0}\right)$ in the integrand of (32). We use the functions defined in Lemma 3.3. Thus we replace $\chi\left(\lambda\left|x-x_{1}\right|\right) Y_{0}\left(\lambda\left|x-x_{1}\right|\right)$ with $F\left(\lambda, x, x_{1}\right)$ and $\chi\left(\lambda\left|y-y_{1}\right|\right) J_{0}(\lambda \mid y-$ $\left.y_{1} \mid\right)$ with $G\left(\lambda, y, y_{1}\right)$ on the left hand side of (32). Therefore the $\lambda$ integral of (32) is equivalent to

$$
\int_{0}^{\infty} e^{i t \lambda^{2}} \lambda \chi(\lambda) \log (\lambda) F\left(\lambda, x, x_{1}\right) G\left(\lambda, y, y_{1}\right) d \lambda .
$$

We integrate by parts once to get

$$
\begin{aligned}
\text { (34) }) & \lesssim|t|^{-1} \int_{0}^{\infty}\left[\log \lambda \chi^{\prime}(\lambda)+\lambda^{-1}\right]\left|F\left(\lambda, x, x_{1}\right)\right|\left|G\left(\lambda, y, y_{1}\right)\right| d \lambda \\
& +|t|^{-1} \int_{0}^{\infty}|\chi(\lambda) \log \lambda|\left|\partial_{\lambda} F\left(\lambda, x, x_{1}\right)\right|\left|G\left(\lambda, y, y_{1}\right)\right| d \lambda \\
& +|t|^{-1} \int_{0}^{\infty}|\chi(\lambda) \log \lambda|\left|F\left(\lambda, x, x_{1}\right)\right|\left|\partial_{\lambda} G\left(\lambda, y, y_{1}\right)\right| d \lambda .
\end{aligned}
$$

There is no boundary term since, by Lemma 3.3 , we have that $F\left(0+, y_{1}, y\right) \lesssim$ $k\left(x, x_{1}\right)$ and $G\left(0, y, y_{1}\right)=0$. From Lemma 3.3 again, we have for any $\tau \in(0,1]$

$$
\text { (35) } \lesssim \int_{0}^{2 \lambda_{1}}\left[\log \lambda+\lambda^{-1}\right] k\left(x, x_{1}\right) \lambda^{\tau}\left\langle y_{1}\right\rangle^{\tau} d \lambda \lesssim\left\langle y_{1}\right\rangle^{\tau} k\left(x, x_{1}\right) .
$$

Taking $\tau=0+$, this term now contributes the following to (32):

$$
\begin{aligned}
& \lesssim|t|^{-1} \int_{\mathbb{R}^{8}} k\left(x, x_{1}\right) v\left(x_{1}\right)\left|D_{1}\left(x_{1}, y_{1}\right)\right| v\left(y_{1}\right)\left\langle y_{1}\right\rangle^{0+}|f(x)||g(y)| d x_{1} d y_{1} d x d y \\
& \lesssim|t|^{-1} \sup _{x \in \mathbb{R}^{2}}\|k(x, \cdot) v(\cdot)\|_{2}\left\|\left|D_{1}\right|\right\|_{2 \rightarrow 2}\|v\|_{L^{2,0+}}\|f\|_{1}\|g\|_{1} \lesssim|t|^{-1} .
\end{aligned}
$$

For the case of (36) and (37), we again note the bounds in Lemma 3.3, and that on the support of $\chi(\lambda),\left|\lambda^{\tau} \log \lambda\right| \lesssim 1$ for any $\tau>0$. The desired bound follows as in (38).

We also need the following bounds, taking care of the contributions of the remaining terms in (29):

Corollary 3.5. For $\mathcal{C}(z)=J_{0}(z)$ or $\mathcal{C}(z)=Y_{0}(z)$, we have the bound

$$
\begin{array}{r}
\mid \int_{\mathbb{R}^{8}} \int_{0}^{\infty} e^{i t \lambda^{2}} \lambda \chi(\lambda) \chi\left(\lambda\left|x-x_{1}\right|\right) \mathcal{C}\left(\lambda\left|x-x_{1}\right|\right) v\left(x_{1}\right) S_{1} D_{1} S_{1}\left(x_{1}, y_{1}\right) v\left(y_{1}\right) \\
\left.\mathcal{C}\left(\lambda\left|y-y_{1}\right|\right) \chi\left(\lambda\left|y-y_{1}\right|\right) d \lambda f(x) g(y) d x_{1} d y_{1} d x d y|\lesssim| t\right|^{-1} .
\end{array}
$$

Proof. Using the notation of Lemma 3.4, we need to bound

$$
\left|\int_{0}^{\infty} e^{i t \lambda^{2}} \lambda \chi(\lambda) F\left(\lambda, x, x_{1}\right) F\left(\lambda, y, y_{1}\right) d \lambda\right|
$$

and the similar term when $F$ is replaced by $G$. This follows easily from one integration by parts and the bounds of Lemma 3.3 as in the previous lemma. 
We now need to bound the resulting terms when one of the Bessel functions is supported on large energies. The following variation of stationary phase from [24] will be useful in the analysis. For completeness we give the proof.

Lemma 3.6. Let $\phi^{\prime}(0)=0$ and $1 \leq \phi^{\prime \prime} \leq C$. Then,

$$
\left|\int_{-\infty}^{\infty} e^{i t \phi(\lambda)} a(\lambda) d \lambda\right| \lesssim \int_{|\lambda|<|t|^{-\frac{1}{2}}}|a(\lambda)| d \lambda+|t|^{-1} \int_{|\lambda|>|t|^{-\frac{1}{2}}}\left(\frac{|a(\lambda)|}{\left|\lambda^{2}\right|}+\frac{\left|a^{\prime}(\lambda)\right|}{|\lambda|}\right) d \lambda .
$$

Proof. Let $\eta \in C_{c}^{\infty}(\mathbb{R})$ be such that $\eta(x)=1$ if $|x|<1$ and $\eta(x)=0$ if $|x|>2$. Let $\eta_{2}(x)=\eta\left(x / 2|t|^{-1 / 2}\right)$. Writing $1=\eta_{2}+\left(1-\eta_{2}\right)$, we rewrite the integral as follows: $\left|\int_{-\infty}^{\infty} e^{i t \phi(\lambda)} a(\lambda) d \lambda\right| \lesssim\left|\int_{-\infty}^{\infty} e^{i t \phi(\lambda)} a(\lambda) \eta_{2}(\lambda) d \lambda\right|+\left|\int_{-\infty}^{\infty} e^{i t \phi(\lambda)} a(\lambda)\left(1-\eta_{2}(\lambda)\right) d \lambda\right|$.

The first term is bounded as in the claim since $\operatorname{supp}\left(\eta_{2}\right)=\left[-|t|^{-\frac{1}{2}},|t|^{-\frac{1}{2}}\right]$. For the second term, we integrate by parts once in $\lambda$ to bound with

$$
|t|^{-1}\left|\int_{-\infty}^{\infty} e^{i t \phi(\lambda)} \frac{d}{d \lambda}\left(\frac{a(\lambda)\left(1-\eta_{2}(\lambda)\right)}{\phi^{\prime}(\lambda)}\right) d \lambda\right|
$$

By Taylor's Theorem,

$$
\phi^{\prime}(\lambda)=\phi^{\prime}(0)+\lambda \phi^{\prime \prime}(c)=\lambda \phi^{\prime \prime}(c) \approx \lambda .
$$

Considering the terms when the derivative acts on $a(\lambda), 1-\eta_{2}(\lambda)$ and $1 / \phi^{\prime}(\lambda)$ finishes the proof.

In addition we have the following high energy analogue of Lemma 3.3 In light of the high energy representations of the Bessel functions (10), recall that for $\mathcal{C} \in$ $\left\{J_{0}, Y_{0}, H_{0}\right\}$,

$$
\mathcal{C}(y) \widetilde{\chi}(y)=e^{i y} \omega_{+}(y)+e^{-i y} \omega_{-}(y), \quad\left|\omega_{ \pm}^{(\ell)}(y)\right| \lesssim\langle y\rangle^{-\frac{1}{2}-\ell} .
$$

Lemma 3.7. Define for $p, q>0$

$$
\widetilde{G}^{ \pm}(\lambda, p, q):=\widetilde{\chi}(\lambda p) \omega_{ \pm}(\lambda p)-e^{ \pm i \lambda(p-q)} \widetilde{\chi}(\lambda q) \omega_{ \pm}(\lambda q),
$$

with $\omega_{ \pm}$as in (10). Then for any $0 \leq \tau \leq 1$ and $\lambda \leq 2 \lambda_{1}$,

$$
\begin{aligned}
& \left|\widetilde{G}^{ \pm}(\lambda, p, q)\right| \lesssim(\lambda|p-q|)^{\tau}\left(\frac{\tilde{\chi}(\lambda p)}{|\lambda p|^{\frac{1}{2}}}+\frac{\tilde{\chi}(\lambda q)}{|\lambda q|^{\frac{1}{2}}}\right), \\
& \left|\partial_{\lambda} \widetilde{G}^{ \pm}(\lambda, p, q)\right| \lesssim|p-q|\left(\frac{\widetilde{\chi}(\lambda p)}{|\lambda p|^{\frac{1}{2}}}+\frac{\widetilde{\chi}(\lambda q)}{|\lambda q|^{\frac{1}{2}}}\right) .
\end{aligned}
$$

Proof. We note first that from (10), we have

$$
\left|\widetilde{G}^{ \pm}(\lambda, p, q)\right| \lesssim \frac{\widetilde{\chi}(\lambda p)}{|\lambda p|^{\frac{1}{2}}}+\frac{\widetilde{\chi}(\lambda q)}{|\lambda q|^{\frac{1}{2}}}
$$

We consider the case of $\widetilde{G}^{+}$; the case of $\widetilde{G}^{-}$is similar. Define the function

$$
b(s):=\tilde{\chi}(s) \omega_{+}(s) .
$$

Using (10), one obtains that for $k=0,1,2, \ldots$,

$$
\left|b^{(k)}(s)\right| \lesssim \widetilde{\chi}(s)|s|^{-\frac{1}{2}-k} .
$$

We now rewrite $\widetilde{G}$ in terms of $b$ :

$$
\widetilde{G}(\lambda, p, q)=b(\lambda p)-b(\lambda q)+\left(1-e^{i \lambda(p-q)}\right) b(\lambda q) .
$$


Note that the absolute value of the last summand is

$$
\lesssim \lambda|p-q| \frac{\tilde{\chi}(\lambda q)}{|\lambda q|^{\frac{1}{2}}}
$$

To estimate the difference between the first two, we assume without loss of generality that $p>q$ and write

$$
|b(\lambda p)-b(\lambda q)|=\left|\int_{\lambda q}^{\lambda p} b^{\prime}(s) d s\right| \lesssim \int_{\lambda q}^{\lambda p} \tilde{\chi}(s)|s|^{-\frac{3}{2}} d s .
$$

In the case $1<\lambda q<\lambda p$, we estimate this integral by

$$
\lambda|p-q| \frac{\tilde{\chi}(\lambda q)}{|\lambda q|^{\frac{3}{2}}} .
$$

In the case $\lambda q<1<\lambda p$, we estimate it as follows:

$$
\begin{aligned}
& \int_{\lambda q}^{\lambda p} \tilde{\chi}(s)|s|^{-\frac{3}{2}} d s \lesssim \tilde{\chi}(\lambda p) \int_{1}^{\lambda p} s^{-3 / 2} d s \lesssim \widetilde{\chi}(\lambda p) \frac{(\lambda p)^{1 / 2}-1}{|\lambda p|^{1 / 2}} \\
& \leq \tilde{\chi}(\lambda p) \frac{(\lambda p)^{1 / 2}-(\lambda q)^{1 / 2}}{|\lambda p|^{1 / 2}} \lesssim \lambda|p-q| \frac{\tilde{\chi}(\lambda p)}{|\lambda p|} .
\end{aligned}
$$

Combining these bounds and interpolating with (41), we obtain the first assertion of the lemma.

We now turn to the derivative. We note that

$$
\begin{aligned}
\partial_{\lambda} \widetilde{G}(\lambda, p, q)= & p b^{\prime}(\lambda p)-q b^{\prime}(\lambda q)+\left(1-e^{i \lambda(p-q)}\right) q b^{\prime}(\lambda q)-i(p-q) e^{i \lambda(p-q)} b(\lambda q) \\
& =\frac{1}{\lambda}\left[b_{1}(\lambda p)-b_{1}(\lambda q)\right]+\frac{1-e^{i \lambda(p-q)}}{\lambda} b_{1}(\lambda q)-i(p-q) e^{i \lambda(p-q)} b(\lambda q),
\end{aligned}
$$

where $b_{1}(s):=s b^{\prime}(s)$ satisfies the same bounds as $b(s)$. Therefore the second assertion of the lemma follows as above.

Lemma 3.8. We have the bound

$$
\begin{array}{r}
\mid \int_{\mathbb{R}^{8}} \int_{0}^{\infty} e^{i t \lambda^{2}} \lambda \chi(\lambda) \log \lambda \chi\left(\lambda\left|x-x_{1}\right|\right) Y_{0}\left(\lambda\left|x-x_{1}\right|\right) v\left(x_{1}\right) S_{1} D_{1} S_{1}\left(x_{1}, y_{1}\right) v\left(y_{1}\right) \\
\left.J_{0}\left(\lambda\left|y-y_{1}\right|\right) \widetilde{\chi}\left(\lambda\left|y-y_{1}\right|\right) d \lambda f(x) g(y) d x_{1} d y_{1} d x d y|\lesssim| t\right|^{-1} .
\end{array}
$$

Proof. Without loss of generality we assume that $t>0$. As in the proof of the previous statements, it suffices to prove that for fixed $x, x_{1}, y, y_{1}$ the $\lambda$-integral is bounded by $k\left(x, x_{1}\right)\left\langle y_{1}\right\rangle t^{-1}$. This power of $\left\langle y_{1}\right\rangle$ necessitates extra decay on the potential to push through the $L^{2}$ mapping bounds as in the previous lemmas. Accordingly, we assume that $v(x) \lesssim\langle x\rangle^{-2-}$ or equivalently that $|V(x)| \lesssim\langle x\rangle^{-4-}$.

Let $p=\max \left(\left|y-y_{1}\right|, 1+|y|\right)$ and $q=\min \left(\left|y-y_{1}\right|, 1+|y|\right)$. Using (133), it suffices to consider

$$
\int_{0}^{\infty} e^{i t \lambda^{2}} \lambda \chi(\lambda) \log \lambda F\left(\lambda, x, x_{1}\right)\left(J_{0}(\lambda p) \widetilde{\chi}(\lambda p)-J_{0}(\lambda q) \widetilde{\chi}(\lambda q)\right) d \lambda
$$

where $F\left(\lambda, x, x_{1}\right)$ is as in Lemma 3.3. The oscillatory term in the definition (10) of $J_{0}$ for large energies will move the stationary point of the phase. Pulling out the 
slower oscillation $e^{ \pm i \lambda q}$, we rewrite this integral as a sum of

$$
\int_{0}^{\infty} e^{i t \phi_{ \pm}(\lambda)} \lambda \chi(\lambda) \log \lambda F\left(\lambda, x, x_{1}\right) \widetilde{G}^{ \pm}(\lambda, p, q) d \lambda
$$

where $\phi_{ \pm}(\lambda)=\lambda^{2} \pm \lambda q t^{-1}$, and $\widetilde{G}$ is from Lemma 3.7. Note that this moves the stationary point of the oscillatory integral to $\lambda_{0}=\mp \frac{q}{2 t}=\mp \frac{\min \left(\left|y-y_{1}\right|, 1+|y|\right)}{2 t}$.

We first consider the contribution of the term with the phase $\phi_{-}(\lambda)$ in which case the critical point satisfies $\lambda_{0} \geq 0$. Let

$$
a(\lambda):=\lambda \chi(\lambda) \log \lambda F\left(\lambda, x, x_{1}\right) \widetilde{G}(\lambda, p, q) .
$$

Using the bounds in Lemma 3.3 and Lemma 3.7 (with $\tau=0+$ ), we have

$$
\begin{aligned}
|a(\lambda)| \lesssim k\left(x, x_{1}\right)\left\langle y_{1}\right\rangle^{0+} \lambda \chi(\lambda)\left(\frac{\tilde{\chi}(\lambda p)}{|\lambda p|^{\frac{1}{2}}}+\frac{\tilde{\chi}(\lambda q)}{|\lambda q|^{\frac{1}{2}}}\right) \text { and } \\
\left|a^{\prime}(\lambda)\right| \lesssim k\left(x, x_{1}\right)\left\langle y_{1}\right\rangle \chi(\lambda)\left(\frac{\tilde{\chi}(\lambda p)}{|\lambda p|^{\frac{1}{2}}}+\frac{\tilde{\chi}(\lambda q)}{|\lambda q|^{\frac{1}{2}}}\right) .
\end{aligned}
$$

We now apply Lemma 3.6 with $a(\lambda)$ as above to bound the $\lambda$-integral in this case by

$$
\int_{\left|\lambda-\lambda_{0}\right|<t^{-1 / 2}}|a(\lambda)| d \lambda+t^{-1} \int_{\left|\lambda-\lambda_{0}\right|>t^{-1 / 2}}\left(\frac{|a(\lambda)|}{\left|\lambda-\lambda_{0}\right|^{2}}+\frac{\left|a^{\prime}(\lambda)\right|}{\left|\lambda-\lambda_{0}\right|}\right) d \lambda .
$$

Using (43), we bound the first integral in (45) by

$$
\int_{\left|\lambda-\lambda_{0}\right|<t^{-1 / 2}}|a(\lambda)| d \lambda \lesssim k\left(x, x_{1}\right)\left\langle y_{1}\right\rangle^{0+} \int_{\left|\lambda-\lambda_{0}\right|<t^{-\frac{1}{2}}} \sqrt{\lambda}\left(\frac{\tilde{\chi}(\lambda p)}{p^{\frac{1}{2}}}+\frac{\tilde{\chi}(\lambda q)}{q^{\frac{1}{2}}}\right) d \lambda .
$$

There are two cases: $\lambda_{0} \gtrsim t^{-\frac{1}{2}}$ and $\lambda_{0} \lesssim t^{-\frac{1}{2}}$. In the former case, on the support of the integral, we have $\lambda \lesssim \lambda_{0}$. Therefore,

$$
\text { (46) } \lesssim k\left(x, x_{1}\right)\left\langle y_{1}\right\rangle^{0+} t^{-\frac{1}{2}} \lambda_{0}^{\frac{1}{2}}\left(p^{-\frac{1}{2}}+q^{-\frac{1}{2}}\right) \lesssim t^{-1} k\left(x, x_{1}\right)\left\langle y_{1}\right\rangle^{0+} \text {. }
$$

In the last inequality, we used $p^{-1} \lambda_{0} \leq q^{-1} \lambda_{0} \leq t^{-1}$. In the latter case, on the support of the integral, we have $\lambda \lesssim t^{-\frac{1}{2}}$, so that

$$
(46) \lesssim k\left(x, x_{1}\right)\left\langle y_{1}\right\rangle^{0+} \int_{0}^{t^{-\frac{1}{2}}} \sqrt{\lambda}\left(\frac{\widetilde{\chi}(\lambda p)}{p^{\frac{1}{2}}}+\frac{\widetilde{\chi}(\lambda q)}{q^{\frac{1}{2}}}\right) d \lambda
$$

For the $\tilde{\chi}(\lambda p)$ term to have any contribution to the integral, we must have that $p^{-1} \lesssim t^{-\frac{1}{2}}$, and similarly for $q^{-1}$. Therefore,

$$
\text { (46) } \lesssim k\left(x, x_{1}\right)\left\langle y_{1}\right\rangle^{0+}\left(p^{-\frac{1}{2}}+q^{-\frac{1}{2}}\right) t^{-\frac{3}{4}} \lesssim k\left(x, x_{1}\right)\left\langle y_{1}\right\rangle^{0+} t^{-1} \text {. }
$$

It suffices to bound the second integral in (45) by $k\left(x, x_{1}\right)\left\langle y_{1}\right\rangle$. We first establish the bounds for the $a(\lambda)$ term and then consider the derivative $a^{\prime}(\lambda)$. 
We have two cases: $\lambda_{0} \ll t^{-\frac{1}{2}}$ and $\lambda_{0} \gtrsim t^{-\frac{1}{2}}$. In the former case, we have $\left|\lambda-\lambda_{0}\right| \approx \lambda$. Thus, using (43), we obtain

$$
\begin{aligned}
\int_{\left|\lambda-\lambda_{0}\right|>t^{-1 / 2}} \frac{|a(\lambda)|}{\left|\lambda-\lambda_{0}\right|^{2}} d \lambda & \lesssim k\left(x, x_{1}\right)\left\langle y_{1}\right\rangle^{0+} \int_{\mathbb{R}} \lambda^{-\frac{3}{2}}\left(\frac{\tilde{\chi}(\lambda p)}{p^{\frac{1}{2}}}+\frac{\tilde{\chi}(\lambda q)}{q^{\frac{1}{2}}}\right) d \lambda \\
& \lesssim k\left(x, x_{1}\right)\left\langle y_{1}\right\rangle^{0+} .
\end{aligned}
$$

In the latter case, we have

$$
\begin{aligned}
& \int_{\left|\lambda-\lambda_{0}\right|>t^{-1 / 2}} \frac{|a(\lambda)|}{\left|\lambda-\lambda_{0}\right|^{2}} d \lambda \\
& \quad \lesssim k\left(x, x_{1}\right)\left\langle y_{1}\right\rangle^{0+} \int_{\left|\lambda-\lambda_{0}\right|>t^{-\frac{1}{2}}} \frac{\lambda^{\frac{1}{2}}}{\left|\lambda-\lambda_{0}\right|^{2}}\left(\frac{\tilde{\chi}(\lambda p)}{p^{\frac{1}{2}}}+\frac{\tilde{\chi}(\lambda q)}{q^{\frac{1}{2}}}\right) d \lambda .
\end{aligned}
$$

Changing the variable $s=\lambda-\lambda_{0}$ and recalling that $p \geq q$, we bound this by

$$
\begin{aligned}
k\left(x, x_{1}\right)\left\langle y_{1}\right\rangle^{0+} q^{-\frac{1}{2}} \int_{|s|>t^{-\frac{1}{2}}} \frac{s^{\frac{1}{2}}+\lambda_{0}^{\frac{1}{2}}}{s^{2}} d s & \lesssim k\left(x, x_{1}\right)\left\langle y_{1}\right\rangle^{0+} q^{-\frac{1}{2}}\left(t^{1 / 4}+t^{1 / 2} \lambda_{0}^{1 / 2}\right) \\
& \lesssim k\left(x, x_{1}\right)\left\langle y_{1}\right\rangle^{0+} .
\end{aligned}
$$

The last inequality follows from the assumption $t^{-1 / 2} \lesssim \lambda_{0}=\frac{q}{2 t}$.

Now, we consider the contribution of $a^{\prime}(\lambda)$. Again we have two cases: $\lambda_{0} \ll t^{-\frac{1}{2}}$ and $\lambda_{0} \gtrsim t^{-\frac{1}{2}}$. In the former case, we have $\left|\lambda-\lambda_{0}\right| \approx \lambda$. Thus, using (44), we obtain

$$
\int_{\left|\lambda-\lambda_{0}\right|>t^{-1 / 2}} \frac{\left|a^{\prime}(\lambda)\right|}{\left|\lambda-\lambda_{0}\right|} d \lambda \lesssim k\left(x, x_{1}\right)\left\langle y_{1}\right\rangle \int_{\mathbb{R}} \lambda^{-\frac{3}{2}}\left(\frac{\tilde{\chi}(\lambda p)}{p^{\frac{1}{2}}}+\frac{\tilde{\chi}(\lambda q)}{q^{\frac{1}{2}}}\right) d \lambda \lesssim k\left(x, x_{1}\right)\left\langle y_{1}\right\rangle .
$$

In the latter case, we have

$$
\begin{aligned}
& \int_{\left|\lambda-\lambda_{0}\right|>t^{-1 / 2}} \frac{\left|a^{\prime}(\lambda)\right|}{\left|\lambda-\lambda_{0}\right|} d \lambda \\
& \quad \lesssim k\left(x, x_{1}\right)\left\langle y_{1}\right\rangle \int_{\left|\lambda-\lambda_{0}\right|>t^{-\frac{1}{2}}} \frac{1}{\left|\lambda-\lambda_{0}\right| \lambda^{\frac{1}{2}}}\left(\frac{\tilde{\chi}(\lambda p)}{p^{\frac{1}{2}}}+\frac{\tilde{\chi}(\lambda q)}{q^{\frac{1}{2}}}\right) d \lambda \\
& \quad \lesssim k\left(x, x_{1}\right)\left\langle y_{1}\right\rangle\left[q^{-\frac{1}{2}} \int_{\left|\lambda-\lambda_{0}\right|>t^{-\frac{1}{2}}} \frac{d \lambda}{\left|\lambda-\lambda_{0}\right|^{\frac{3}{2}}}+\int_{\mathbb{R}} \frac{d \lambda}{\lambda^{\frac{3}{2}}}\left(\frac{\tilde{\chi}(\lambda p)}{p^{\frac{1}{2}}}+\frac{\tilde{\chi}(\lambda q)}{q^{\frac{1}{2}}}\right)\right] \\
& \quad \lesssim k\left(x, x_{1}\right)\left\langle y_{1}\right\rangle\left[q^{-\frac{1}{2}} t^{1 / 4}+1\right] \lesssim k\left(x, x_{1}\right)\left\langle y_{1}\right\rangle .
\end{aligned}
$$

The second inequality follows from $\frac{1}{\left|\lambda-\lambda_{0}\right| \lambda^{1 / 2}} \leq \frac{1}{\left|\lambda-\lambda_{0}\right|^{3 / 2}}+\frac{1}{\lambda^{3 / 2}}$, and the last one from the assumption $t^{-1 / 2} \lesssim \lambda_{0}=\frac{q}{2 t}$.

When considering the phase $\phi_{+}(\lambda)=\lambda^{2}+\lambda q t^{-1}$, integration by parts suffices to obtain the desired bound since the phase has no critical points on $(0, \infty)$. We have

$$
\left|\int_{0}^{\infty} e^{i t \phi_{+}(\lambda)} a(\lambda) d \lambda\right| \lesssim t^{-1}\left(\int_{0}^{\infty} \frac{|a(\lambda)|}{\left|\phi_{+}^{\prime}(\lambda)\right|^{2}} d \lambda+\int_{0}^{\infty} \frac{\left|a^{\prime}(\lambda)\right|}{\left|\phi_{+}^{\prime}(\lambda)\right|} d \lambda\right) .
$$

Using (43), (44), and $\phi_{+}^{\prime}(\lambda) \geq 2 \lambda$, we bound the right hand side by

$$
\lesssim k\left(x, x_{1}\right)\left\langle y_{1}\right\rangle t^{-1} \int_{0}^{\infty} \lambda^{-\frac{3}{2}}\left(\frac{\tilde{\chi}(\lambda p)}{p^{\frac{1}{2}}}+\frac{\tilde{\chi}(\lambda q)}{q^{\frac{1}{2}}}\right) d \lambda \lesssim k\left(x, x_{1}\right)\left\langle y_{1}\right\rangle t^{-1} .
$$


When switching roles of $\tilde{\chi}$ and $\chi$ in (42), we note that from (10) the high energy Bessel function representation holds for $Y_{0}(y)$ as well. The proof will move along the same line, with $G\left(\lambda, y, y_{1}\right)$ in place of $\widetilde{G}\left(\lambda, y, y_{1}\right)$ and using $\widetilde{G}\left(\lambda, x, x_{1}\right)$ in place of $F\left(\lambda, x, x_{1}\right)$. The case when both Bessel functions are $Y_{0}$ or $J_{0}$ is similar:

Corollary 3.9. For $\mathcal{C}(z)=J_{0}(z)$ or $\mathcal{C}(z)=Y_{0}(z)$, we have the bound

$$
\begin{array}{r}
\mid \int_{\mathbb{R}^{8}} \int_{0}^{\infty} e^{i t \lambda^{2}} \lambda \chi(\lambda) \chi\left(\lambda\left|x-x_{1}\right|\right) \mathcal{C}\left(\lambda\left|x-x_{1}\right|\right) v\left(x_{1}\right) S_{1} D_{1} S_{1}\left(x_{1}, y_{1}\right) v\left(y_{1}\right) \\
\left.\mathcal{C}\left(\lambda\left|y-y_{1}\right|\right) \widetilde{\chi}\left(\lambda\left|y-y_{1}\right|\right) d \lambda f(x) g(y) d x_{1} d y_{1} d x d y|\lesssim| t\right|^{-1}
\end{array}
$$

We now consider the case when both Bessel functions are supported on high energies. For this we will use the first line of (29):

Lemma 3.10. We have the bound

$$
\begin{aligned}
& \mid \int_{\mathbb{R}^{8}} \int_{0}^{\infty} e^{i t \lambda^{2}} \lambda \chi(\lambda) h^{ \pm}(\lambda) \widetilde{\chi}\left(\lambda\left|x-x_{1}\right|\right) H_{0}^{ \pm}\left(\lambda\left|x-x_{1}\right|\right) v\left(x_{1}\right) S_{1} D_{1} S_{1}\left(x_{1}, y_{1}\right) v\left(y_{1}\right) \\
& \left.H_{0}^{ \pm}\left(\lambda\left|y-y_{1}\right|\right) \widetilde{\chi}\left(\lambda\left|y-y_{1}\right|\right) d \lambda f(x) g(y) d x_{1} d y_{1} d x d y|\lesssim| t\right|^{-1} .
\end{aligned}
$$

Proof. Again we assume that $t>0$. Recall (9):

$$
H_{0}^{ \pm}(z) \widetilde{\chi}(z)=e^{ \pm i z} \omega_{ \pm}(z)
$$

with $\left|\omega_{ \pm}^{(\ell)}(z)\right| \lesssim(1+|z|)^{-\frac{1}{2}-\ell}$. As in Lemma 3.8 we need to use the auxiliary function $\widetilde{G}$. Denote $p_{1}=\max \left(\left|x-x_{1}\right|, 1+|x|\right), p_{2}=\min \left(\left|x-x_{1}\right|, 1+|x|\right), q_{1}=\max (\mid y-$ $\left.y_{1}|, 1+| y \mid\right)$ and $q_{2}=\min \left(\left|y-y_{1}\right|, 1+|y|\right)$. Without loss of generality, $p_{1}, p_{2}, q_{1}, q_{2}>$ 0 . We note that by (33), we can replace $H_{0}^{ \pm}\left(\lambda\left|x-x_{1}\right|\right)$ with $\widetilde{G}\left(\lambda, p_{1}, p_{2}\right)$ (and similarly replace $\left.H_{0}^{ \pm}\left(\lambda\left|y-y_{1}\right|\right)\right)$ as in Lemma 3.7. This changes the phase with

$$
\phi_{ \pm}(\lambda)=\lambda^{2} \pm \lambda \frac{p_{2}+q_{2}}{t} .
$$

We first consider $\phi_{-}(\lambda)$, which has a stationary point at $\lambda_{0}=\frac{p_{2}+q_{2}}{2 t}>0$. We apply Lemma 3.6 with

$$
a(\lambda)=\lambda \chi(\lambda) h^{ \pm}(\lambda) \widetilde{G}\left(\lambda, p_{1}, p_{2}\right) \widetilde{G}\left(\lambda, q_{1}, q_{2}\right) .
$$

Using the bounds in Lemma 3.7 (with $\tau=0+$ for $\widetilde{G}\left(\lambda, p_{1}, p_{2}\right)$ and $\tau=0$ for the other), we have

$$
\begin{aligned}
|a(\lambda)| \lesssim\left\langle x_{1}\right\rangle^{0+} \chi(\lambda)\left(\frac{\tilde{\chi}\left(\lambda p_{1}\right)}{p_{1}^{1 / 2}}+\frac{\tilde{\chi}\left(\lambda p_{2}\right)}{p_{2}^{1 / 2}}\right)\left(\frac{\tilde{\chi}\left(\lambda q_{1}\right)}{q_{1}^{1 / 2}}+\frac{\tilde{\chi}\left(\lambda q_{2}\right)}{q_{2}^{1 / 2}}\right) \text { and } \\
\left|a^{\prime}(\lambda)\right| \lesssim\left\langle x_{1}\right\rangle\left\langle y_{1}\right\rangle \frac{\chi(\lambda)}{\lambda}\left(\frac{\tilde{\chi}\left(\lambda p_{1}\right)}{p_{1}^{1 / 2}}+\frac{\tilde{\chi}\left(\lambda p_{2}\right)}{p_{2}^{1 / 2}}\right)\left(\frac{\tilde{\chi}\left(\lambda q_{1}\right)}{q_{1}^{1 / 2}}+\frac{\tilde{\chi}\left(\lambda q_{2}\right)}{q_{2}^{1 / 2}}\right) .
\end{aligned}
$$

Let $\tau=2 \max \left(\lambda_{0}, t^{-1 / 2}\right)$. Since $\tilde{\chi}$ is a non-decreasing function supported on $[1, \infty)$ and $\lambda \leq \tau$ on the support of the integral, we have the bound

$$
\begin{aligned}
& \int_{\left|\lambda-\lambda_{0}\right|<t^{-1 / 2}}|a(\lambda)| d \lambda \lesssim\left\langle x_{1}\right\rangle^{0+} t^{-1 / 2}\left(\frac{\tilde{\chi}\left(\tau p_{1}\right)}{p_{1}^{1 / 2}}+\frac{\widetilde{\chi}\left(\tau p_{2}\right)}{p_{2}^{1 / 2}}\right)\left(\frac{\widetilde{\chi}\left(\tau q_{1}\right)}{q_{1}^{1 / 2}}+\frac{\tilde{\chi}\left(\tau q_{2}\right)}{q_{2}^{1 / 2}}\right) \\
& \lesssim\left\langle x_{1}\right\rangle^{0+} t^{-1 / 2} \tau \lesssim\left\langle x_{1}\right\rangle^{0+} t^{-1},
\end{aligned}
$$


if $\tau=2 t^{-1 / 2}$. On the other hand, if $\tau=2 \lambda_{0}$, we consider the contributions of the products of $\tilde{\chi}$ 's more carefully. Consider the contribution of

$$
\frac{\tilde{\chi}\left(\tau p_{i}\right) \tilde{\chi}\left(\tau q_{j}\right)}{p_{i}^{1 / 2} q_{j}^{1 / 2}}
$$

to (52). This term is zero unless $p_{i} \gtrsim 1 / \lambda_{0}$ and $q_{j} \gtrsim 1 / \lambda_{0}$. Therefore, using $p_{1} \geq p_{2}$ and $q_{1} \geq q_{2}$, we have

$$
\frac{p_{2}+q_{2}}{p_{i} q_{j}} \leq \frac{p_{i}+q_{j}}{p_{i} q_{j}}=\frac{1}{p_{i}}+\frac{1}{q_{j}} \lesssim \lambda_{0}=\frac{p_{2}+q_{2}}{2 t} .
$$

Therefore, $p_{i} q_{j} \gtrsim t$, and we can estimate the contribution of each product to (52) by $\left\langle x_{1}\right\rangle^{0+} t^{-1}$.

For the portion of $a(\lambda)$ supported on $\left|\lambda-\lambda_{0}\right|>t^{-\frac{1}{2}}$, we note that if $\lambda_{0} \ll t^{-\frac{1}{2}}$, then $\left|\lambda-\lambda_{0}\right| \approx \lambda$ so that

$$
\begin{aligned}
\int_{\left|\lambda-\lambda_{0}\right|>t^{-\frac{1}{2}}} \frac{|a(\lambda)|}{\left|\lambda-\lambda_{0}\right|^{2}} d \lambda \lesssim\left\langle x_{1}\right\rangle^{0+} \sum_{i, j=1}^{2} \int_{\mathbb{R}} \frac{\tilde{\chi}\left(\lambda p_{i}\right)}{p_{i}^{1 / 2}} \frac{\tilde{\chi}\left(\lambda q_{j}\right)}{q_{j}^{1 / 2}} \frac{d \lambda}{\lambda^{2}} \\
\lesssim\left\langle x_{1}\right\rangle^{0+} \sum_{i, j=1}^{2}\left(\int_{\mathbb{R}} \frac{\tilde{\chi}\left(\lambda p_{i}\right)^{2}}{p_{i}} \frac{d \lambda}{\lambda^{2}}\right)^{\frac{1}{2}}\left(\int_{\mathbb{R}} \frac{\tilde{\chi}\left(\lambda q_{j}\right)^{2}}{q_{j}} \frac{d \lambda}{\lambda^{2}}\right)^{\frac{1}{2}} \lesssim\left\langle x_{1}\right\rangle^{0+} .
\end{aligned}
$$

On the other hand, if $\lambda_{0} \gtrsim t^{-\frac{1}{2}}$, we have

$$
\int_{\left|\lambda-\lambda_{0}\right|>t^{-\frac{1}{2}}} \frac{|a(\lambda)|}{\left|\lambda-\lambda_{0}\right|^{2}} d \lambda \lesssim\left\langle x_{1}\right\rangle^{0+} \sum_{i, j=1}^{2}\left(p_{i} q_{j}\right)^{-\frac{1}{2}} \int_{\left|\lambda-\lambda_{0}\right|>t^{-\frac{1}{2}}} \frac{\tilde{\chi}\left(\lambda p_{i}\right) \widetilde{\chi}\left(\lambda q_{j}\right)}{\left|\lambda-\lambda_{0}\right|^{2}} d \lambda .
$$

Fix $i, j$ and let $m=\min \left(p_{i}, q_{j}\right)$. We have two cases: $\lambda_{0} \ll 1 / m$ and $\lambda_{0} \gtrsim 1 / m$. In the former case, we note that $\left|\lambda-\lambda_{0}\right| \gtrsim 1 / m$ on the support of the cutoffs. Therefore,

$$
\begin{aligned}
& \left(p_{i} q_{j}\right)^{-\frac{1}{2}} \int_{\left|\lambda-\lambda_{0}\right|>t^{-\frac{1}{2}}} \frac{\tilde{\chi}\left(\lambda p_{i}\right) \tilde{\chi}\left(\lambda q_{j}\right)}{\left|\lambda-\lambda_{0}\right|^{2}} d \lambda \\
& \quad \lesssim\left(p_{i} q_{j}\right)^{-\frac{1}{2}} \int_{\left|\lambda-\lambda_{0}\right| \gtrsim 1 / m} \frac{d \lambda}{\left|\lambda-\lambda_{0}\right|^{2}} \lesssim\left(p_{i} q_{j}\right)^{-\frac{1}{2}} m \leq 1 .
\end{aligned}
$$

In the latter case, using (53) we conclude that $p_{i} q_{j} \gtrsim t$. This implies the desired bound by ignoring the cutoffs in the integral.

We now turn to the term in Lemma 3.6 that involves $a^{\prime}(\lambda)$. Using (51), we have

$$
\begin{gathered}
\int_{\left|\lambda-\lambda_{0}\right|>t^{-1 / 2}} \frac{\left|a^{\prime}(\lambda)\right|}{\left|\lambda-\lambda_{0}\right|} d \lambda \lesssim\left\langle x_{1}\right\rangle\left\langle y_{1}\right\rangle \sum_{i, j=1}^{2}\left(p_{i} q_{j}\right)^{-\frac{1}{2}} \int_{\left|\lambda-\lambda_{0}\right|>t^{-1 / 2}} \frac{\tilde{\chi}\left(\lambda p_{i}\right) \widetilde{\chi}\left(\lambda q_{j}\right)}{\lambda\left|\lambda-\lambda_{0}\right|} d \lambda \\
\lesssim\left\langle x_{1}\right\rangle\left\langle y_{1}\right\rangle \sum_{i, j=1}^{2}\left(p_{i} q_{j}\right)^{-\frac{1}{2}}\left[\int_{\left|\lambda-\lambda_{0}\right|>t^{-1 / 2}} \frac{\tilde{\chi}\left(\lambda p_{i}\right) \widetilde{\chi}\left(\lambda q_{j}\right)}{\lambda^{2}} d \lambda\right. \\
\left.+\int_{\left|\lambda-\lambda_{0}\right|>t^{-1 / 2}} \frac{\tilde{\chi}\left(\lambda p_{i}\right) \widetilde{\chi}\left(\lambda q_{j}\right)}{\left|\lambda-\lambda_{0}\right|^{2}} d \lambda\right] .
\end{gathered}
$$

The required bounds for each of these terms appeared above in the bound for $a(\lambda) /\left|\lambda-\lambda_{0}\right|^{2}$ integral. This establishes the desired bound for the phase $\phi_{-}$. For the case of $\phi_{+}$, integration by parts and the bounds on $a(\lambda)$ and $a^{\prime}(\lambda)$ suffice. We leave the details to the reader. 
With these estimates established, we are ready to prove Proposition 3.2

Proof of Proposition 3.2. Lemmas 3.4, 3.8, 3.10 and Corollaries 3.5, 3.9 bound each term of (29), as desired.

We now turn to the terms involving $S S_{1} D_{1} S_{1}$ and $S_{1} D_{1} S_{1} S$ in Corollary 2.7

Proposition 3.11. The contribution of the terms $S S_{1} D_{1} S_{1}, S_{1} D_{1} S_{1} S$ and $Q D_{0} Q$ in Corollary 2.7 to (2) satisfies (28). More explicitly, we have the bound

$$
\begin{gathered}
\mid \int_{\mathbb{R}^{8}} \int_{0}^{\infty} e^{i t \lambda^{2}} \lambda \chi(\lambda) R_{0}^{ \pm}\left(\lambda^{2}\right)\left(x, x_{1}\right) v\left(x_{1}\right) S S_{1} D_{1} S_{1}\left(x_{1}, y_{1}\right) v\left(y_{1}\right) \\
\left.R_{0}^{ \pm}\left(\lambda^{2}\right)\left(y, y_{1}\right) d \lambda f(x) g(y) d x_{1} d y_{1} d x d y|\lesssim| t\right|^{-1} .
\end{gathered}
$$

The same bound holds when $S S_{1} D_{1} S_{1}$ is replaced by $S_{1} D_{1} S_{1} S$ or by $Q D_{0} Q$.

Proof. The $Q D_{0} Q$ term can be handled as in Proposition 3.2. It is in fact easier since there is no $\log (\lambda)$ term.

The other terms are somehow different since they have a projection orthogonal to $v$ only on one side. Therefore, one can use (33) only on one side. However, since there is no $\log (\lambda)$ term, the bounds established in Lemmas 3.4, 3.8, and 3.10 go through. For instance, to establish the bound

$$
\begin{array}{r}
\mid \int_{\mathbb{R}^{8}} \int_{0}^{\infty} e^{i t \lambda^{2}} \lambda \chi(\lambda) \chi\left(\lambda\left|x-x_{1}\right|\right) Y_{0}\left(\lambda\left|x-x_{1}\right|\right) v\left(x_{1}\right) S S_{1} D_{1} S_{1}\left(x_{1}, y_{1}\right) v\left(y_{1}\right) \\
\left.J_{0}\left(\lambda\left|y-y_{1}\right|\right) \chi\left(\lambda\left|y-y_{1}\right|\right) d \lambda f(x) g(y) d x_{1} d y_{1} d x d y|\lesssim| t\right|^{-1},
\end{array}
$$

we can use $G\left(\lambda, y, y_{1}\right)$ in place of $J_{0}\left(\lambda\left|y-y_{1}\right|\right)$. After an integration by parts the boundary terms vanish since $G\left(\lambda, y, y_{1}\right) \rightarrow 0$ as $\lambda \rightarrow 0$, and the $\lambda$-integral can be bounded by

$$
\begin{aligned}
& t^{-1} \int_{0}^{\infty}\left|\partial_{\lambda}\left(\chi(\lambda) \chi\left(\lambda\left|x-x_{1}\right|\right) Y_{0}\left(\lambda\left|x-x_{1}\right|\right) G\left(\lambda, y, y_{1}\right)\right)\right| d \lambda \\
\lesssim & t^{-1}\left\langle y_{1}\right\rangle^{\tau}\left(1+\log ^{-}\left(\left|x-x_{1}\right|\right)\right) \int_{0}^{1}(1+|\log (\lambda)|) \lambda^{\tau-1} d \lambda \lesssim t^{-1}\left\langle y_{1}\right\rangle^{\tau}\left(1+\log ^{-}\left(\left|x-x_{1}\right|\right)\right) .
\end{aligned}
$$

Here we used the bounds for $G$ from Lemma 3.3, the bounds (7) and (8), and the following estimate:

$$
\chi(\lambda) \chi\left(\lambda\left|x-x_{1}\right|\right) \log \left(\lambda\left|x-x_{1}\right|\right) \lesssim(1+|\log (\lambda)|)\left(1+\log ^{-}\left(\left|x-x_{1}\right|\right)\right) .
$$

This estimate follows easily by considering the cases $\left|x-x_{1}\right|<1$ and $\left|x-x_{1}\right|>1$ separately.

When we have $S_{1} D_{1} S_{1} S$ instead, we must use $F\left(\lambda, x, x_{1}\right)$ instead of $Y_{0}\left(\lambda\left|x-x_{1}\right|\right)$, and the boundary terms are now controlled by $|t|^{-1} k\left(x, x_{1}\right)$ as in Lemma 3.3 . The other cases when both projections are onto low energies can be handled similarly.

The case when $\chi$ is replaced with $\tilde{\chi}$ on both sides can be handled as in Lemma 15 from [24] since the argument there does not make use of the projections orthogonal to $v$.

Similarly, in the case when $\chi$ is replaced with $\tilde{\chi}$ on the side which does not have a projection orthogonal to $v$, the proof of Lemma 14 from 24] applies. 
It remains to prove that

$$
\begin{array}{r}
\mid \int_{\mathbb{R}^{8}} \int_{0}^{\infty} e^{i t \lambda^{2}} \lambda \chi(\lambda) \chi\left(\lambda\left|x-x_{1}\right|\right) Y_{0}\left(\lambda\left|x-x_{1}\right|\right) v\left(x_{1}\right) S S_{1} D_{1} S_{1}\left(x_{1}, y_{1}\right) v\left(y_{1}\right) \\
\left.J_{0}\left(\lambda\left|y-y_{1}\right|\right) \widetilde{\chi}\left(\lambda\left|y-y_{1}\right|\right) d \lambda f(x) g(y) d x_{1} d y_{1} d x d y|\lesssim| t\right|^{-1} .
\end{array}
$$

Since $S$ is not orthogonal to $v$, we cannot replace $\chi Y_{0}$ with $F$. However, we can replace $\widetilde{\chi} J_{0}$ with $\widetilde{G}$ shifting the critical point of the $\lambda$-integral as in the proof of Lemma 3.8. The argument in the proof of that lemma relies on the bounds

$$
\left|\log (\lambda) F\left(\lambda, x, x_{1}\right)\right| \lesssim \log (\lambda) k\left(x, x_{1}\right), \quad\left|\partial_{\lambda}\left(\log (\lambda) F\left(\lambda, x, x_{1}\right)\right)\right| \lesssim k\left(x, x_{1}\right) \log (\lambda) \lambda^{-1} .
$$

Since we don't have an additional $\log (\lambda)$ in (55), it suffices to note that $\chi(\lambda) \chi(\lambda \mid x-$ $\left.x_{1} \mid\right) Y_{0}\left(\lambda\left|x-x_{1}\right|\right)$ satisfies similar bounds as in (56) (with $1+\left|\log ^{-}\left(\mid x-x_{1}\right)\right|$ instead of $k\left(x, x_{1}\right)$; cf. (54) $)$.

The terms arising from $h_{ \pm}(\lambda)^{-1} S$ and $h_{ \pm}(\lambda)^{-1} S S_{1} D_{1} S_{1} S$ are handled in Lemma 17 in [24], which we restate below for completeness.

Proposition 3.12 (24]). The contribution of the terms $h_{ \pm}(\lambda)^{-1} S$ and $h_{ \pm}(\lambda)^{-1} S S_{1} D_{1} S_{1} S$ in Corollary 2.7 to (2) satisfies (28). More explicitly, we have the bound

$$
\left|\int_{0}^{\infty} e^{i t \lambda^{2}} \lambda \chi(\lambda)\left\langle\left[\frac{R_{0}^{+}\left(\lambda^{2}\right) v S v R_{0}^{+}\left(\lambda^{2}\right)}{h_{+}(\lambda)}-\frac{R_{0}^{-}\left(\lambda^{2}\right) v S v R_{0}^{-}\left(\lambda^{2}\right)}{h_{-}(\lambda)}\right] f, g\right\rangle d \lambda\right| \lesssim|t|^{-1} \text {. }
$$

$A$ similar bound holds if we replace $S$ with $S S_{1} D_{1} S_{1} S$.

Finally, the following proposition (Lemma 18 from 24]) takes care of the contribution of the error term in Corollary 2.7 to (2).

Proposition $3.13([24])$. Assume that $\Phi(\lambda)$ is an absolutely bounded operator on $L^{2}\left(\mathbb{R}^{2}\right)$ that satisfies $\Phi(\lambda)=\mathcal{O}_{1}\left(\lambda^{\frac{1}{2}}\right)$. We have the bound

$$
\begin{array}{r}
\mid \int_{\mathbb{R}^{8}} \int_{0}^{\infty} e^{i t \lambda^{2}} \lambda \chi(\lambda) R_{0}^{ \pm}\left(\lambda^{2}\right)\left(x, x_{1}\right) v\left(x_{1}\right) \Phi(\lambda)\left(x_{1}, y_{1}\right) v\left(y_{1}\right) R_{0}^{ \pm}\left(\lambda^{2}\right)\left(y, y_{1}\right) d \lambda \\
\left.f(x) g(y) d x_{1} d y_{1} d x d y|\lesssim| t\right|^{-1} .
\end{array}
$$

\section{RESONANCES OF THE SECOND AND THIRD KIND}

We now consider the evolution in the case of a p-wave resonance and/or an eigenvalue at zero. Recall that this case is characterized by the non-invertibility of $T_{1}=S_{1} T P T S_{1}$. To obtain resolvent expansions around zero, we need to invert the operator $B_{ \pm}$, (20). The expansions in this section are considerably more complicated than those in the case of a resonance of the first kind given in Proposition 2.6.

Recall the operators $S_{2}, S_{3}, T_{2}$, and $T_{3}$ from Definition 2.3. With a slight abuse of the notation, we define $D_{1}:=\left(T_{1}+S_{2}\right)^{-1}=\left(S_{1} T P T S_{1}+S_{2}\right)^{-1}$ as an operator on $S_{1} L^{2}\left(\mathbb{R}^{2}\right)$. We define $D_{2}:=\left(T_{2}+S_{3}\right)^{-1}=\left(S_{2} v G_{1} v S_{2}+S_{3}\right)^{-1}$ on $S_{2} L^{2}\left(\mathbb{R}^{2}\right)$, and we will also use $D_{2}$ for $T_{2}^{-1}$ when $T_{2}$ is invertible, i.e. when $S_{2}=0$. We also define $D_{3}:=T_{3}^{-1}=\left(S_{3} v G_{2} v S_{3}\right)^{-1}$ on $S_{3} L^{2}\left(\mathbb{R}^{2}\right)$. 
Proposition 4.1. Assume that $v(x) \lesssim\langle x\rangle^{-3-}$. Then, $B_{ \pm}$is invertible on $S_{1} L^{2}\left(\mathbb{R}^{2}\right)$. In the case of a resonance of the second kind, we have

$$
B_{ \pm}^{-1}=\frac{S_{2} D_{2} S_{2}}{g_{1}^{ \pm}(\lambda)}+O\left(\lambda^{-2}|\log \lambda|^{-2}\right)
$$

where $g_{1}^{ \pm}(\lambda)$ is as in Lemma 2.2 .

In the case of a resonance of the third kind, we have

$$
B_{ \pm}^{-1}=\frac{S_{3} D_{3} S_{3}}{\lambda^{2}}+\frac{S_{2} \mathcal{D} S_{2}}{g_{1}^{ \pm}(\lambda)}+O\left(\lambda^{-2}|\log \lambda|^{-2}\right) .
$$

Here

$$
\begin{aligned}
\mathcal{D}= & D_{2}+S_{3} D_{3} S_{3} v G_{2} v S_{2} D_{2} S_{2} v G_{2} v S_{3} D_{3} S_{3}-S_{3} D_{3} S_{3} v G_{2} v S_{2} D_{2} \\
& -D_{2} S_{2} v G_{2} v S_{3} D_{3} S_{3} .
\end{aligned}
$$

Proof. We give the proof for the case of the "+" superscripts and subscripts and omit them from the proof. Recall the definition (20) of $B$ :

$$
B=S_{1}-S_{1}\left(M(\lambda)+S_{1}\right)^{-1} S_{1} .
$$

First we repeat the expansion that we obtained in Proposition 2.6 by keeping better track of the error term. Using Lemma 2.5, the identity

$$
A^{-1}(\lambda)\left[\mathbb{1}+M_{0} A^{-1}(\lambda)\right]^{-1}=A^{-1}(\lambda)-A^{-1}(\lambda) M_{0} A^{-1}(\lambda)\left[\mathbb{1}+M_{0} A^{-1}(\lambda)\right]^{-1},
$$

and the definition (23) of $A^{-1}(\lambda)$, we obtain

$$
B=S_{1}-S_{1}\left(h(\lambda)^{-1} S+Q D_{0} Q\right) S_{1}+E(\lambda)
$$

where

$$
\begin{aligned}
& E(\lambda)=S_{1} A^{-1}(\lambda) M_{0}(\lambda) A^{-1}(\lambda)\left[\mathbb{1}+M_{0}(\lambda) A^{-1}(\lambda)\right]^{-1} S_{1} \\
& =S_{1} A^{-1}(\lambda) M_{0}(\lambda) A^{-1}(\lambda) S_{1}-S_{1} A^{-1}(\lambda)\left[M_{0}(\lambda) A^{-1}(\lambda)\right]^{2}\left[\mathbb{1}+M_{0}(\lambda) A^{-1}(\lambda)\right]^{-1} S_{1} .
\end{aligned}
$$

Since $v(x) \lesssim\langle x\rangle^{-3-}$, by Lemma 2.2 , we have $M_{0}=\mathcal{O}_{1}\left(\lambda^{2-}\right)$. Also using $A^{-1}(\lambda)=$ $\mathcal{O}_{1}(1)$ (from (23)), we conclude that

$$
E(\lambda)=\mathcal{O}_{1}\left(\lambda^{2-}\right) \text {. }
$$

Recall that $S_{1} D_{0}=D_{0} S_{1}=S_{1}$. Further, from the definition (22) of $S$ and the fact that $S_{1} P=P S_{1}=0$, we obtain $S_{1} S S_{1}=S_{1} T P T S_{1}$. Therefore

$$
B=-h(\lambda)^{-1} S_{1} S S_{1}+E(\lambda)=-h(\lambda)^{-1} S_{1} T P T S_{1}+E(\lambda) .
$$

In the case of a resonance of the second kind (unlike the case of a resonance of the first kind), the leading term $T_{1}=S_{1} T P T S_{1}$ above is not invertible. We will invert the operator

$$
B_{1}:=-h(\lambda) B=T_{1}-h(\lambda) E(\lambda)
$$

by using Lemma 2.4. Let $S_{2}$ be the Riesz projection onto the kernel of $T_{1}$, and let $D_{1}:=\left(T_{1}+S_{2}\right)^{-1}$. We have

$$
\begin{aligned}
\left(B_{1}+S_{2}\right)^{-1} & =\left[T_{1}+S_{2}-h(\lambda) E(\lambda)\right]^{-1}=D_{1}\left[\mathbb{1}-h(\lambda) E(\lambda) D_{1}\right]^{-1} \\
& =D_{1}+D_{1} h(\lambda) E(\lambda) D_{1}+D_{1}\left[h(\lambda) E(\lambda) D_{1}\right]^{2}\left[\mathbb{1}-h(\lambda) E(\lambda) D_{1}\right]^{-1} \\
& =D_{1}+O\left(\lambda^{2-}\right) .
\end{aligned}
$$


By Lemma 2.4, $B_{1}$ is invertible if

$$
B_{2}:=S_{2}-S_{2}\left(B_{1}+S_{2}\right)^{-1} S_{2}
$$

is invertible on $S_{2} L^{2}$. Using (63), the identities $S_{2} D_{1}=D_{1} S_{2}=S_{2}$, and the definition (60) of $E(\lambda)$, we have

$$
\begin{aligned}
B_{2} & =-h(\lambda) S_{2} E(\lambda) S_{2}-S_{2}\left[h(\lambda) E(\lambda) D_{1}\right]^{2}\left[\mathbb{1}-h(\lambda) E(\lambda) D_{1}\right]^{-1} S_{2} \\
& =-h(\lambda) S_{2} A^{-1}(\lambda) M_{0}(\lambda) A^{-1}(\lambda) S_{2}+E_{1}(\lambda),
\end{aligned}
$$

where

$$
\begin{aligned}
E_{1}(\lambda)=h(\lambda) S_{2} A^{-1}(\lambda)\left[M_{0}(\lambda) A^{-1}(\lambda)\right]^{2}\left[\mathbb{1}+M_{0}(\lambda) A^{-1}(\lambda)\right]^{-1} S_{2} \\
-S_{2}\left[h(\lambda) E(\lambda) D_{1}\right]^{2}\left[\mathbb{1}-h(\lambda) E(\lambda) D_{1}\right]^{-1} S_{2}
\end{aligned}
$$

We now claim that

$$
P T S_{2}=S_{2} T P=0 \text {. }
$$

To see this, note that since $S_{2}, T$ and $P$ are self-adjoint and since $S_{2}$ is the projection onto the kernel of $S_{1} T P T S_{1}$, we have

$$
\left\langle P T S_{2} f, P T S_{2} f\right\rangle=\left\langle S_{2} T P T S_{2} f, f\right\rangle=\left\langle S_{2} S_{1} T P T S_{1} S_{2} f, f\right\rangle=0 .
$$

Therefore,

$$
A^{-1}(\lambda) S_{2}=S_{2} A^{-1}(\lambda)=S_{2} .
$$

Using this and the expansion (15) of $M_{0}$, we rewrite $B_{2}$ as

$$
\begin{aligned}
B_{2} & =-h(\lambda) g_{1}(\lambda) S_{2} v G_{1} v S_{2}-h(\lambda) \lambda^{2} S_{2} v G_{2} v S_{2}-h(\lambda) S_{2} M_{1}(\lambda) S_{2}+E_{1}(\lambda) \\
& =:-h(\lambda) g_{1}(\lambda)\left[T_{2}+\lambda^{2} g_{1}^{-1}(\lambda) S_{2} v G_{2} v S_{2}+E_{2}(\lambda)\right] .
\end{aligned}
$$

By Lemma 2.2, we have $M_{0}=\mathcal{O}_{1}\left(\lambda^{2-}\right)$ and $h(\lambda) S_{2} M_{1}(\lambda) S_{2}=\mathcal{O}_{1}\left(\lambda^{2-}\right)$. Also using $A^{-1}(\lambda)=\mathcal{O}_{1}(1)$ and (61), we conclude that $E_{1}(\lambda)=\mathcal{O}_{1}\left(\lambda^{4-}\right)$. This yields that $E_{2}(\lambda)=\mathcal{O}_{1}\left(\lambda^{2-}\right)$. In the case of a resonance of the second kind, the leading term is invertible. Therefore, for small $\lambda$,

$$
B_{2}^{-1}=-\frac{\left[T_{2}+\lambda^{2} g_{1}^{-1}(\lambda) S_{2} v G_{2} v S_{2}+E_{2}(\lambda)\right]^{-1}}{h(\lambda) g_{1}(\lambda)}=-\frac{D_{2}}{h(\lambda) g_{1}(\lambda)}+O\left(\lambda^{-2}|\log \lambda|^{-3}\right) .
$$

Using Lemma 2.4, (63), (67), and the identities $S_{2} D_{1}=D_{1} S_{2}=S_{2}$, we have

$$
\begin{aligned}
B^{-1} & =-h(\lambda) B_{1}^{-1}=-h(\lambda)\left[\left(B_{1}+S_{2}\right)^{-1}+\left(B_{1}+S_{2}\right)^{-1} S_{2} B_{2}^{-1} S_{2}\left(B_{1}+S_{2}\right)^{-1}\right] \\
& =\frac{S_{2} D_{2} S_{2}}{g_{1}(\lambda)}+O\left((\lambda \log \lambda)^{-2}\right) .
\end{aligned}
$$

In the case of a resonance of the third kind, the leading term in $B_{2}$ is not invertible. Analogously, we will invert the operator

$$
B_{3}=-h^{-1}(\lambda) g_{1}^{-1}(\lambda) B_{2}=T_{2}+\lambda^{2} g_{1}^{-1}(\lambda) S_{2} v G_{2} v S_{2}+E_{2}(\lambda)
$$


by using Lemma 2.4 Let $S_{3}$ be the Riesz projection onto the kernel of $T_{2}$, and let $D_{2}:=\left(T_{2}+S_{3}\right)^{-1}$. We have

$$
\begin{aligned}
\left(B_{3}+S_{3}\right)^{-1}= & {\left[T_{2}+S_{3}+\lambda^{2} g_{1}^{-1}(\lambda) S_{2} v G_{2} v S_{2}+E_{2}(\lambda)\right]^{-1} } \\
= & D_{2}-\lambda^{2} g_{1}^{-1}(\lambda) D_{2} S_{2} v G_{2} v S_{2} D_{2}+\lambda^{4} g_{1}^{-2}(\lambda) D_{2}\left[S_{2} v G_{2} v S_{2} D_{2}\right]^{2} \\
& +O\left(|\log \lambda|^{-3}\right) .
\end{aligned}
$$

In the second line we used the definition of $g_{1}(\lambda)$ in Lemma 2.2 and the estimate on $E_{2}(\lambda)$.

By Lemma 2.4 $B_{3}$ is invertible if

$$
B_{4}:=S_{3}-S_{3}\left(B_{3}+S_{3}\right)^{-1} S_{3}
$$

is invertible on $S_{3} L^{2}$. Using (69), the identities $S_{3} D_{2}=D_{2} S_{3}=S_{3}$, and $T_{3}=$ $S_{3} v G_{2} v S_{3}$, we have

$$
B_{4}=\lambda^{2} g_{1}^{-1}(\lambda) T_{3}-\lambda^{4} g_{1}^{-2}(\lambda) S_{3}\left[S_{2} v G_{2} v S_{2} D_{2}\right]^{2} S_{3}+O\left(|\log \lambda|^{-3}\right) .
$$

Since $T_{3}$ is always invertible (see Section 4 of 15$]$ ), $B_{4}$ is invertible for small $\lambda$, and we have

$$
B_{4}^{-1}=\lambda^{-2} g_{1}(\lambda) D_{3}+\widetilde{D}_{2}+O\left(|\log \lambda|^{-1}\right),
$$

where $\widetilde{D}_{2}=D_{3} S_{3} v G_{2} v S_{2} D_{2} S_{2} v G_{2} v S_{3} D_{3}$.

Using this, Lemma 2.4. and (69), we have

$$
\begin{aligned}
B_{2}^{-1}= & -\frac{1}{h(\lambda) g_{1}(\lambda)} B_{3}^{-1} \\
= & -\frac{1}{h(\lambda) g_{1}(\lambda)}\left[\left(B_{3}+S_{3}\right)^{-1}+\left(B_{3}+S_{3}\right)^{-1} S_{3} B_{4}^{-1} S_{3}\left(B_{3}+S_{3}\right)^{-1}\right] \\
= & -\frac{S_{3} D_{3} S_{3}}{\lambda^{2} h(\lambda)}-\frac{D_{2}+S_{3} \widetilde{D}_{2} S_{3}-S_{3} D_{3} S_{3} v G_{2} v S_{2} D_{2}-D_{2} S_{2} v G_{2} v S_{3} D_{3} S_{3}}{h(\lambda) g_{1}(\lambda)} \\
& +O\left(\lambda^{-2}|\log \lambda|^{-3}\right) .
\end{aligned}
$$

Using this (instead of (67)) for $B^{-1}$ in (68) yields the assertion of the proposition.

Corollary 4.2. Assume that $v(x) \lesssim\langle x\rangle^{-3-}$. Then, in the case of a resonance of the second kind, we have

$$
\begin{aligned}
M^{ \pm}(\lambda)^{-1}=\frac{S_{2} D_{2} S_{2}}{g_{1}^{ \pm}(\lambda)}+Q \Gamma_{1}^{ \pm}(\lambda) Q+Q \Gamma_{2}^{ \pm}(\lambda) & +\Gamma_{3}^{ \pm}(\lambda) Q+\Gamma_{4}^{ \pm}(\lambda) \\
& +\left(M^{ \pm}(\lambda)+S_{1}\right)^{-1}+\mathcal{O}_{1}\left(\lambda^{2-}\right)
\end{aligned}
$$

where $\Gamma_{i}^{ \pm}, i=1,2,3,4$, are absolutely bounded operators on $L^{2}\left(\mathbb{R}^{2}\right)$ with $\Gamma_{1}^{ \pm}(\lambda)=$ $O\left(\lambda^{-2}(\log \lambda)^{-2}\right), \Gamma_{2}^{ \pm}(\lambda), \Gamma_{3}^{ \pm}(\lambda)=O\left(\lambda^{-2}(\log \lambda)^{-3}\right)$, and $\Gamma_{4}^{ \pm}(\lambda)=O\left(\lambda^{-2}(\log \lambda)^{-4}\right)$.

In the case of a resonance of the third kind, we have

$$
\begin{aligned}
M^{ \pm}(\lambda)^{-1}=\frac{S_{3} D_{3} S_{3}}{\lambda^{2}}+\frac{S_{2} \mathcal{D} S_{2}}{g_{1}^{ \pm}(\lambda)}+Q \Gamma_{1}^{ \pm}(\lambda) Q & +Q \Gamma_{2}^{ \pm}(\lambda)+\Gamma_{3}^{ \pm}(\lambda) Q+\Gamma_{4}^{ \pm}(\lambda) \\
& +\left(M^{ \pm}(\lambda)+S_{1}\right)^{-1}+\mathcal{O}_{1}\left(\lambda^{2-}\right)
\end{aligned}
$$


where $\mathcal{D}$ is as in Proposition 4.1 and $\Gamma_{i}$ are absolutely bounded operators on $L^{2}\left(\mathbb{R}^{2}\right)$. These operators are distinct from the $\Gamma_{i}$ in the case of a resonance of the second kind but satisfy the same size estimates.

Proof. For a resonance of the second kind, combining Proposition 4.1 with Lemma 2.4 and Lemma 2.5 (taking the decay condition on $v$ into account), we have

$$
\begin{aligned}
M(\lambda)^{-1} & =\left(M(\lambda)+S_{1}\right)^{-1}+\left(M(\lambda)+S_{1}\right)^{-1} S_{1} B^{-1} S_{1}\left(M(\lambda)+S_{1}\right)^{-1} \\
& =\left(M(\lambda)+S_{1}\right)^{-1}+\frac{1}{g_{1}(\lambda)}\left(A(\lambda)^{-1}+\mathcal{O}_{1}\left(\lambda^{2-}\right)\right) S_{2} D_{2} S_{2}\left(A(\lambda)^{-1}+\mathcal{O}_{1}\left(\lambda^{2-}\right)\right) \\
& +\left(A(\lambda)^{-1}+\mathcal{O}_{1}\left(\lambda^{2-}\right)\right) O\left((\lambda \log \lambda)^{-2}\right)\left(A(\lambda)^{-1}+\mathcal{O}_{1}\left(\lambda^{2-}\right)\right) .
\end{aligned}
$$

Using (66) and the definition (23) of $A^{-1}$, we obtain

$$
\begin{aligned}
M(\lambda)^{-1} & =\frac{S_{2} D_{2} S_{2}}{g_{1}(\lambda)}+\left(M(\lambda)+S_{1}\right)^{-1}+\mathcal{O}_{1}\left(\lambda^{2-}\right) \\
& +\left(Q D_{0} Q+O\left(|\log \lambda|^{-1}\right)\right) O\left((\lambda \log \lambda)^{-2}\right)\left(Q D_{0} Q+O\left(|\log \lambda|^{-1}\right)\right) .
\end{aligned}
$$

The second line leads to four different terms yielding (70).

For the case of a resonance of the third kind, the statement follows similarly using formula (58) for $B^{-1}$.

We now consider the dispersive estimates in the case when $H$ has a p-wave resonance at zero energy. Comparing (70) to the expansion in Corollary 2.7, we note that many of the terms in the expansion for resonances of the second kind are in the expansion for resonances of the first kind. Accordingly, it suffices to establish the estimates for the contributions of the terms

$$
\frac{S_{2} D_{2} S_{2}}{g_{1}^{ \pm}(\lambda)}+Q \Gamma_{1}(\lambda) Q+Q \Gamma_{2}(\lambda)+\Gamma_{3}(\lambda) Q+\Gamma_{4}(\lambda) .
$$

We start with the following.

Lemma 4.3. We have the bound

$$
\left|\int_{0}^{\infty} e^{i t \lambda^{2}} \lambda \chi(\lambda)\left\langle\left[\frac{R_{0}^{+}\left(\lambda^{2}\right) v S_{2} D_{2} S_{2} v R_{0}^{+}\left(\lambda^{2}\right)}{g_{1}^{+}(\lambda)}-\frac{R_{0}^{-}\left(\lambda^{2}\right) v D_{2} v R_{0}^{-}\left(\lambda^{2}\right)}{g_{1}^{-}(\lambda)}\right] f, g\right\rangle d \lambda\right| \lesssim 1 .
$$

Proof. We note that we must exploit some cancellation between the ' + ' and '-' terms. Recall that $H_{0}^{ \pm}(y)=J_{0}(y) \pm i Y_{0}(y)$ and the definition of $g_{1}^{ \pm}(\lambda)$ in Lemma 2.2 give us

$$
\begin{aligned}
\frac{R_{0}^{+}\left(\lambda^{2}\right) R_{0}^{+}\left(\lambda^{2}\right)}{g_{1}^{+}(\lambda)} & -\frac{R_{0}^{-}\left(\lambda^{2}\right) R_{0}^{-}\left(\lambda^{2}\right)}{g_{1}^{-}(\lambda)}=\frac{J_{0}(\lambda p) J_{0}(\lambda q)-Y_{0}(\lambda p) Y_{0}(\lambda q)}{\lambda^{2}\left[\left(\log \lambda+c_{1}\right)^{2}+c_{2}^{2}\right]} \\
& +\frac{\left(J_{0}(\lambda p) Y_{0}(\lambda q)+Y_{0}(\lambda p) J_{0}(\lambda q)\right)\left(\log \lambda+c_{1}\right)}{\lambda^{2}\left[\left(\log \lambda+c_{1}\right)^{2}+c_{2}^{2}\right]} .
\end{aligned}
$$

We again must use the cut-offs $\chi$ and $\tilde{\chi}$ and consider the different cases depending on the supports of the resolvents. Let us first consider the case when both resolvents are supported on low energy. Contribution of the first term in (73) satisfies the required bound since $J_{0}=O(1)$, and $\frac{1}{\lambda(\log \lambda)^{2}}$ is integrable on $\left[0, \lambda_{1}\right]$. Since the other terms have additional powers $\log \lambda$ in the numerator, we need to use (33) (recall that $S_{2} \leq Q$ ). 
Consider the contribution of the second term in (73). Using (33), we replace $\chi Y_{0}$ with $F(\lambda, \cdot, \cdot)$, and using Lemma 3.3 , we obtain the bound

$$
\begin{aligned}
\left|\int_{0}^{\infty} \chi(\lambda) \frac{F\left(\lambda, x, x_{1}\right) F\left(\lambda, y, y_{1}\right)}{\lambda\left[\left(\log \lambda+c_{1}\right)^{2}+c_{2}^{2}\right]} d \lambda\right| & \lesssim k\left(x, x_{1}\right) k\left(y, y_{1}\right) \int_{0}^{1} \frac{1}{\lambda(\log \lambda)^{2}} d \lambda \\
& \lesssim k\left(x, x_{1}\right) k\left(y, y_{1}\right) .
\end{aligned}
$$

The mixed $J_{0}$ and $Y_{0}$ terms in the second part of (173) are bounded similarly using $\left|G\left(\lambda, x, x_{1}\right)\right| \lesssim \lambda^{0+}\left\langle x_{1}\right\rangle^{0+}$.

An analysis as in (38) shows that these terms satisfy the desired bound (72).

When one or both of the Bessel functions is supported on high energies, we use the functions $\widetilde{G}(\lambda, p, q)$ from Lemma 3.7. The bound $|\widetilde{G}(\lambda, p, q)| \lesssim \lambda^{0+}|p-q|^{0+}$ suffices for obtaining the required bound. The details are left to the reader.

Lemma 4.4. For $\mathcal{C}_{i}(z)=J_{0}(z)$ or $Y_{0}(z)$ for $i=1,2$, we have the bound

$$
\begin{gathered}
\mid \int_{\mathbb{R}^{8}} \int_{0}^{\infty} e^{i t \lambda^{2}} \lambda \chi(\lambda) \mathcal{C}_{1}\left(\lambda\left|x-x_{1}\right|\right) v\left(x_{1}\right) Q \Gamma_{1}(\lambda) Q\left(x_{1}, y_{1}\right) v\left(y_{1}\right) \\
\mathcal{C}_{2}\left(\lambda\left|y-y_{1}\right|\right) d \lambda f(x) g(y) d x_{1} d y_{1} d x d y \mid \lesssim 1 .
\end{gathered}
$$

Proof. Unlike Lemma 4.3 we do not need to use any cancellation between the ' + ' and '-' terms. We consider the terms that arise when both $\mathcal{C}_{1}$ and $\mathcal{C}_{2}$ are supported on small energies. Consider

$$
\int_{0}^{\infty} e^{i t \lambda^{2}} \lambda \chi(\lambda) \chi(\lambda p) \mathcal{C}_{1}(\lambda p) v Q \Gamma_{1}(\lambda) Q v \chi(\lambda q) \mathcal{C}_{2}(\lambda q) d \lambda
$$

where $p=\left|x-x_{1}\right|, q=\left|y-y_{1}\right|$. In the worst case when $\mathcal{C}_{1}=\mathcal{C}_{2}=Y_{0}$, using (33), we replace $\chi Y_{0}$ with $F$ to obtain

$$
\begin{aligned}
\mid \int_{0}^{1} & \lambda F\left(\lambda, x, x_{1}\right) \Gamma_{1}(\lambda) F\left(\lambda, y, y_{1}\right) d \lambda \mid \\
& \lesssim\left|\int_{0}^{1} \frac{F\left(\lambda, x, x_{1}\right) F\left(\lambda, y, y_{1}\right)}{\lambda(\log \lambda)^{2}} d \lambda\right| \sup _{0<\lambda<\lambda_{1}}\left|\lambda^{2}(\log \lambda)^{2} \Gamma_{1}(\lambda)\right| \\
& \lesssim k\left(x, x_{1}\right) k\left(y, y_{1}\right) \sup _{0<\lambda<\lambda_{1}}\left|\lambda^{2}(\log \lambda)^{2} \Gamma_{1}(\lambda)\right| .
\end{aligned}
$$

The last line follows from Lemma 3.3. Since $\sup _{0<\lambda<\lambda_{1}}\left|\lambda^{2}(\log \lambda)^{2} \Gamma_{1}(\lambda)\right|$ defines a bounded operator on $L^{2}\left(\mathbb{R}^{2}\right)$ (by Corollary 4.2), we are done. The other low energy terms are similar, using $G$ instead of $F$ from Lemma 3.3

For the large energies, we note that the argument runs in a similar manner. Using $\tilde{\chi}(y)\left(\left|J_{0}(y)\right|+\left|Y_{0}(y)\right|\right) \lesssim 1$ and an argument as in (74), it easily follows that the integral is bounded, as desired.

The following modification of Lemma 4.4 is necessary for the other $\Gamma_{i}(\lambda)$ terms.

Corollary 4.5. For $\mathcal{C}_{i}(z)=J_{0}(z)$ or $Y_{0}(z)$ for $i=1,2$, we have the bound

$$
\left|\int_{\mathbb{R}^{8}} \int_{0}^{\infty} e^{i t \lambda^{2}} \lambda \chi(\lambda) \mathcal{C}_{1}\left(\lambda\left|x-x_{1}\right|\right) v\left(x_{1}\right) Q \Gamma_{2}(\lambda)\left(x_{1}, y_{1}\right) v\left(y_{1}\right) \mathcal{C}_{2}\left(\lambda\left|y-y_{1}\right|\right) d \lambda d x_{1} d y_{1}\right|
$$

$\lesssim 1$.

The same bounds hold when $Q \Gamma_{2}(\lambda)$ is replaced by $\Gamma_{3}(\lambda) Q$ or $\Gamma_{4}(\lambda)$. 
Proof. We repeat the analysis of Lemma 4.4. Consider the case when both $\mathcal{C}_{i}(\lambda \cdot)$ are supported on low energies and both are $Y_{0}$. We note that when $\lambda<1$, using (54), we have

$$
\left|Y_{0}(\lambda p) \chi(\lambda p)\right| \lesssim(1+|\log \lambda|)\left(1+\log ^{-} p\right) .
$$

Using this and replacing $\chi Y_{0}$ with $F$ on one side, we obtain the bound

$$
\begin{aligned}
\int_{0}^{\lambda_{1}} & \frac{\left|F\left(\lambda, x, x_{1}\right)\right|\left(1+\log ^{-} q\right)}{\lambda|\log \lambda|^{2}} d \lambda \sup _{0<\lambda<\lambda_{1}}\left|\lambda^{2}(\log \lambda)^{3} \Gamma_{2}(\lambda)\right| \\
& \lesssim k\left(x, x_{1}\right) k\left(y, y_{1}\right) \sup _{0<\lambda<\lambda_{1}}\left|\lambda^{2}(\log \lambda)^{3} \Gamma_{2}(\lambda)\right| .
\end{aligned}
$$

The same bound holds for $\Gamma_{3}(\lambda) Q$. For the contribution of $\Gamma_{4}(\lambda)$, we have

$$
\begin{aligned}
& \left|\int_{0}^{\infty} \lambda \chi(\lambda) Y_{0}(\lambda p) \Gamma_{4}(\lambda) Y_{0}(\lambda q) d \lambda\right| \\
& \lesssim \int_{0}^{1} \frac{(1+|\log \lambda|)\left(1+\log ^{-} p\right)(1+|\log \lambda|)\left(1+\log ^{-} q\right)}{\lambda|\log \lambda|^{4}} d \lambda \sup _{0<\lambda<\lambda_{1}}\left|\lambda^{2}(\log \lambda)^{4} \Gamma_{4}(\lambda)\right| \\
& \lesssim k\left(x, x_{1}\right) k\left(y, y_{1}\right) \sup _{0<\lambda<\lambda_{1}}\left|\lambda^{2}(\log \lambda)^{4} \Gamma_{4}(\lambda)\right| .
\end{aligned}
$$

The other cases are similar.

When one of the $\mathcal{C}_{i}(\lambda \cdot)$ is supported on high energies, the analysis is less delicate. The required bound follows from $\tilde{\chi}(y)\left(\left|J_{0}(y)\right|+\left|Y_{0}(y)\right|\right) \lesssim 1$.

This completes the proof in the case of a resonance of the second kind.

We note that the above bounds in Lemma 4.4 and Corollary 4.5 also hold for the $\Gamma_{i}$ term in (71). Thus for a resonance of the third kind, it suffices to consider the leading $\lambda^{-2}$ term in (71). Noting (29) and the fact that the kernel of $D_{3}$ is realvalued, the following lemma completes the proof. In the next section we will prove that $G_{0} v S_{3} D_{3} S_{3} v G_{0}$ is the projection onto the zero eigenspace whose contribution disappears since we project away from the zero eigenspace. We will ignore this issue in the proof below since the eigenfunctions are bounded functions and hence the projection onto the zero eigenspace satisfies the desired bound, and since removing this operator requires more decay from the potential; see Section 6 .

Lemma 4.6. We have the bound

$$
\left|\int_{\mathbb{R}^{8}} \int_{0}^{\infty} e^{i t \lambda^{2}} \lambda \chi(\lambda) J_{0}\left(\lambda\left|x-x_{1}\right|\right) v\left(x_{1}\right) \frac{S_{3} D_{3} S_{3}}{\lambda^{2}} v\left(y_{1}\right) Y_{0}\left(\lambda\left|y-y_{1}\right|\right) d \lambda d x_{1} d y_{1}\right| \lesssim 1 .
$$

Proof. We provide a sketch of the proof. Due to similarities to previous proofs, we leave the details to the reader. We again consider the case when the Bessel functions are supported on low energy first. Accordingly, we wish to control

$$
\begin{aligned}
\mid \int_{0}^{\infty} e^{i t \lambda^{2}} \lambda \chi & (\lambda) \chi(\lambda p) J_{0}(\lambda p) v\left(x_{1}\right) \frac{D_{3}}{\lambda^{2}} v\left(y_{1}\right) \chi(\lambda q) Y_{0}(\lambda q) d \lambda \mid \\
& \lesssim \mid \int_{0}^{\infty} \chi(\lambda) \frac{G\left(\lambda, x, x_{1}\right) F\left(\lambda, y, y_{1}\right)}{\lambda} d \lambda \lesssim\left\langle x_{1}\right\rangle^{\tau} k\left(y, y_{1}\right),
\end{aligned}
$$

where we used (33) and Lemma 3.3 with any $\tau>0$. 
For the case when one function is supported on high energy, we have

$$
\begin{aligned}
\left|\int_{0}^{\infty} e^{i t \lambda^{2}} \lambda \chi(\lambda) \widetilde{\chi}(\lambda p) J_{0}(\lambda p) v\left(x_{1}\right) \frac{D_{3}}{\lambda^{2}} v\left(y_{1}\right) \chi(\lambda q) Y_{0}(\lambda q) d \lambda\right| \\
\qquad \mid \int_{0}^{\infty} \chi(\lambda) \frac{\widetilde{G}\left(\lambda, x, x_{1}\right) F\left(\lambda, y, y_{1}\right)}{\lambda} d \lambda \lesssim\left\langle x_{1}\right\rangle^{\tau} k\left(y, y_{1}\right) .
\end{aligned}
$$

Similarly one uses $\widetilde{G}\left(\lambda, y, y_{1}\right)$ instead of $F\left(\lambda, y, y_{1}\right)$ if we have $\widetilde{\chi}(\lambda q)$.

When both functions are supported on high energy, we have

$$
\begin{aligned}
\mid \int_{0}^{\infty} e^{i t \lambda^{2}} \lambda & \chi(\lambda) \widetilde{\chi}(\lambda p) J_{0}(\lambda p) v\left(x_{1}\right) \frac{D_{3}}{\lambda^{2}} v\left(y_{1}\right) \widetilde{\chi}(\lambda q) Y_{0}(\lambda q) d \lambda \mid \\
& \lesssim \mid \int_{0}^{\infty} \chi(\lambda) \frac{\widetilde{G}\left(\lambda, x, x_{1}\right) \widetilde{G}\left(\lambda, y, y_{1}\right)}{\lambda} d \lambda \lesssim\left\langle x_{1}\right\rangle^{\tau}\left\langle y_{1}\right\rangle^{\tau} .
\end{aligned}
$$

An analysis as in (38) finishes the proof.

We are now ready to prove the main theorem of this section.

Theorem 4.7. Let $V: \mathbb{R}^{2} \rightarrow \mathbb{R}$ be such that $|V(x)| \lesssim\langle x\rangle^{-\beta}$ for some $\beta>6$. Further assume that $H=-\Delta+V$ has a resonance of the second or third kind at zero energy. Then there is a time dependent operator $F_{t}$ such that

$$
\sup _{t}\left\|F_{t}\right\|_{L^{1} \rightarrow L^{\infty}} \lesssim 1, \quad\left\|K_{\lambda_{1}}-F_{t}\right\|_{L^{1} \rightarrow L^{\infty}} \lesssim|t|^{-1}, \quad|t|>1 .
$$

Proof. If we denote the terms that arise from the contribution of the terms in the first lines of (70) and (71) as $F_{t}$, Lemmas 4.3, 4.4, and 4.6 and Corollary 4.5 show that

$$
\sup _{t}\left\|F_{t}\right\|_{L^{1} \rightarrow L^{\infty}} \lesssim 1
$$

As the remaining terms in (70) and (71) are identical in form to those that arise in the proof of Theorem 3.1, we can use the bounds from the previous section to establish the theorem.

Finally, we note that Theorem 1.1 follows directly from Theorem 1.3. Theorem 3.1. Theorem 4.7, and the first remark following Definition 2.3.

\section{SpeCtral Structure of $-\Delta+V$ AT Zero ENERGy}

In this section, we prove some of the claims made in the remark following Definition 2.3. In particular, we show the relationship between the spectral subspaces $S_{i} L^{2}\left(\mathbb{R}^{2}\right)$ for $1=1,2,3$ and distributional solutions to $H \psi=0$.

Let $w=U v$. First we characterize $S_{1} L^{2}$.

Lemma 5.1. If $v \lesssim\langle x\rangle^{-1-}$ and if $\phi \in S_{1} L^{2}$, then $\phi=w \psi$ where $\psi \in L^{\infty}, H \psi=0$ in the sense of distributions, and

$$
\psi=c_{0}-G_{0} v \phi, \quad c_{0}=\frac{1}{\|V\|_{L^{1}}}\langle v, T \phi\rangle .
$$

Moreover, if $v \lesssim\langle x\rangle^{-2-}$, then $\psi-c_{0}=-G_{0} v \phi \in L^{p}$ for any $p \in(2, \infty]$. 
Proof. Since $\phi \in S_{1} L^{2}$, we have $Q \phi=\phi$. Using this and $P=I-Q$, we have

$$
0=Q T Q \phi=Q T \phi=T \phi-P T \phi=U \phi+v G_{0} v \phi-P T \phi .
$$

Thus,

$$
\phi=-w G_{0} v \phi+U P T \phi=-w G_{0} v \phi+w c_{0}=w \psi .
$$

Also note that since $v(x) \lesssim\langle x\rangle^{-1-}$ and $\phi \in L^{2}$, we have $-\Delta G_{0}(v \phi)=v \phi$. Therefore, we see that $H \psi=0$ by taking the distributional derivative.

Now we prove that $\psi \in L^{\infty}$. The boundedness on $B(0,4)$ is clear. To see that $\psi$ is bounded for $|x|>4$, use $P \phi=0$ to obtain

$$
\begin{aligned}
G_{0} v \phi(x) & =-\frac{1}{2 \pi} \int[\log (|x-y|)-\log (|x|)] v(y) \phi(y) d y \\
& =-\frac{1}{2 \pi} \int \log \left(\frac{|x-y|}{|x|}\right) v(y) \phi(y) d y .
\end{aligned}
$$

The bound follows by using the inequality (for $|x|>4$ )

$$
\left|\log \left(\frac{|x-y|}{|x|}\right)\right| \lesssim 1+\log (\langle y\rangle)+\log ^{-}(|x-y|) .
$$

Note that this only requires that $v(x) \lesssim\langle x\rangle^{-1-}$.

The final statement follows if we can prove that $G_{0} v \phi=O\left(|x|^{-1}\right)$ for large $x$. To see this, write

$$
\begin{aligned}
G_{0} v \phi(x) & =-\frac{1}{2 \pi} \int \log \left(\frac{|x-y|}{|x|}\right) v(y) \phi(y) d y \\
& =\int_{|y|>|x| / 2} \log \left(\frac{|x-y|}{|x|}\right) v(y) \phi(y) d y+\int_{|y|<|x| / 2} \log \left(\frac{|x-y|}{|x|}\right) v(y) \phi(y) d y .
\end{aligned}
$$

The first integral can be estimated by

$$
\int_{|y|>|x| / 2}\left[1+\log (\langle y\rangle)+\log ^{-}(|x-y|)\right] \frac{|y||v(y) \phi(y)|}{|x|} d y=O(1 /|x|) .
$$

On the other hand, the bound for the second integral follows from

$$
\left|\log \left(\frac{|x-y|}{|x|}\right)\right|=\left|\log \left(1+\frac{|x-y|-|x|}{|x|}\right)\right|=O\left(\frac{|| x-y|-| x||}{|x|}\right)=O(|y| /|x|) .
$$

Lemma 5.2. Let $v \lesssim\langle x\rangle^{-2-}$. Assume that the function $\psi=c+\Lambda_{1}+\Lambda_{2}$, with $\Lambda_{1} \in$ $L^{p}$ for some $p \in(2, \infty)$ and $\Lambda_{2} \in L^{2}$, solves $H \psi=0$ in the sense of distributions. Then $\phi=w \psi \in S_{1} L^{2}$, and we have $\psi=c-G_{0} v \phi, c=\frac{1}{\|V\|_{L^{1}}}\langle v, T \phi\rangle$. In particular, by the previous claim, $\psi-c \in L^{p}$ for any $p \in(2, \infty]$.

Proof. Since $H \psi=0$, we have

$$
\Delta \psi=V \psi=v \phi
$$

This easily implies that $\int v(y) \phi(y) d y=0$; see [15, Lemma 6.4]. Thus $\phi \in Q L^{2}$.

Now consider the function $\psi+G_{0} v \phi$. By the calculation above, we see that $\Delta\left(\psi+G_{0} v \phi\right)=0$. Since $\psi+G_{0} v \phi \in L^{2}+L^{\infty}$ (by assumption and the proof of the previous claim), we see that it has to be a constant. Thus

$$
\psi=c-G_{0} v \phi .
$$


Using this, we have

$$
T Q \phi=T \phi=U \phi+v G_{0} v \phi=U \phi-v \psi+c v=U \phi-U \phi+c v=c v,
$$

and hence $Q T Q \phi=0$, and $\phi \in S_{1} L^{2}$. Finally, this implies that $c=\frac{1}{\|V\|_{L^{1}}}\langle v, T \phi\rangle$.

Note that Lemma 5.1 and Lemma 5.2 imply that all zero eigenfunctions are bounded. We now characterize $S_{2} L^{2}$.

Lemma 5.3. Assume that $|v(x)| \lesssim\langle x\rangle^{-3-}$. Then $\phi=w \psi \in S_{2} L^{2}$ if and only if $\psi \in L^{p}$, for all $p \in(2, \infty]$ (or equivalently $c_{0}=0$ ).

Proof. Recall that $S_{2} \leq S_{1}$ is a projection onto the kernel of $S_{1} T P T S_{1}$. We have (since $S_{1} \phi=\phi$ )

$$
S_{1} T P T S_{1} \phi=0 \Longrightarrow\|P T \phi\|^{2}=\langle T P T \phi, \phi\rangle=0,
$$

and hence $c_{0}=0$ and $\psi \in L^{p}$ for $p>2$.

On the other hand, if $\psi \in L^{p}$ for $p>2$, we have $c_{0}=0$. This implies that $P T \phi=P T S_{1} \phi=0$, and hence $S_{1} T P T S_{1} \phi=0$.

Lemma 5.4. If $v \lesssim\langle x\rangle^{-3-}$, then the kernel of the operator $S_{3} v G_{2} v S_{3}$ on $S_{3} L^{2}$ is trivial.

Proof. Given $f$ in the kernel of $S_{3} v G_{2} v S_{3}$, we have

$$
\int_{\mathbb{R}^{2}} f(y) v(y) d y=0, \text { and } S_{2} v G_{1} v S_{2} f=0,
$$

since $f \in S_{3} L^{2} \subset Q L^{2}$.

Also note that the expansion we used for $R_{0}^{+}\left(\lambda^{2}\right)$ in the proof of Lemma 2.2 gives that

$R_{0}^{+}\left(\lambda^{2}\right)(x, y)=g^{+}(\lambda)+G_{0}(x, y)+g_{1}^{+}(\lambda) G_{1}(x, y)+\lambda^{2} G_{2}(x, y)+O\left(\lambda^{2+}|x-y|^{2+}\right)$.

This and the assumption $v \lesssim\langle x\rangle^{-3-}$ imply that

$$
\begin{aligned}
0 & =\left\langle S_{3} v G_{2} v S_{3} f, f\right\rangle=\left\langle v G_{2} v f, f\right\rangle \\
& =\lim _{\lambda \rightarrow 0}\left\langle\frac{R_{0}^{+}\left(\lambda^{2}\right)-g^{+}(\lambda)-G_{0}-g_{1}^{+}(\lambda) G_{1}}{\lambda^{2}} v f, v f\right\rangle .
\end{aligned}
$$

Now, using (79), we have

$$
\begin{aligned}
0 & =\left\langle v G_{2} v f, f\right\rangle=\lim _{\lambda \rightarrow 0}\left\langle\frac{R_{0}\left(\lambda^{2}\right)-G_{0}}{\lambda^{2}} v f, v f\right\rangle \\
& =\lim _{\lambda \rightarrow 0} \frac{1}{\lambda^{2}} \int_{\mathbb{R}^{2}}\left(\frac{1}{4 \pi^{2} \xi^{2}+\lambda^{2}}-\frac{1}{4 \pi^{2} \xi^{2}}\right)|\widehat{v f}|^{2} d \xi \\
& =\frac{1}{4 \pi^{2}} \lim _{\lambda \rightarrow 0} \int_{\mathbb{R}^{2}} \frac{1}{\xi^{2}\left(4 \pi^{2} \xi^{2}+\lambda^{2}\right)}|\widehat{v f}|^{2} d \xi=\frac{1}{(2 \pi)^{4}} \int_{\mathbb{R}^{2}} \frac{|\widehat{v f}|^{2}}{\xi^{4}} d \xi,
\end{aligned}
$$

where we used the monotone convergence theorem in the last step. By the assumptions on $v$ and $f, v f \in L^{1}$, and hence $v f=0$. We also know that $f \in S_{1} L^{2}$ and hence $f=w \psi$, which implies that $f=0$. This establishes the invertibility of the operator $S_{3} v G_{2} v S_{3}$ on $S_{3} L^{2}$. 
Further, we have the identity for any $f \in S_{3} L^{2}$,

$$
\begin{aligned}
\left\langle v G_{2} v f, f\right\rangle & =\frac{1}{(2 \pi)^{4}} \int_{\mathbb{R}^{2}} \frac{|\widehat{v f}|^{2}}{\xi^{4}} d \xi=\frac{1}{(2 \pi)^{4}}\left\langle\frac{\widehat{v f}(\xi)}{\xi^{2}}, \frac{\widehat{v f}(\xi)}{\xi^{2}}\right\rangle \\
& =\left\langle(-\Delta)^{-1} v f,(-\Delta)^{-1} v f\right\rangle \\
& =\left\langle G_{0} v f, G_{0} v f\right\rangle .
\end{aligned}
$$

Lemma 5.5. Assume that $v(x) \lesssim\langle x\rangle^{-3-}$. Then $\phi=w \psi \in S_{3} L^{2}$ if and only if $\psi \in L^{2}$ with $H \psi=0$.

Proof. For $\phi \in S_{3} L^{2} \subset S_{1} L^{2}$, we proved above that $\phi=w \psi$ with

$$
H \psi=0, \quad \psi=c_{0}-G_{0} v \phi, \quad c_{0}=\frac{1}{\|V\|_{L^{1}}}\langle v, T \phi\rangle .
$$

Also note that $c_{0}=0$ by (78) since $\phi \in S_{2} L^{2}$. Therefore, using (80), we have

$$
\|\psi\|_{2}^{2}=\langle\psi, \psi\rangle=\left\langle G_{0} v \phi, G_{0} v \phi\right\rangle=\left\langle v G_{2} v \phi, \phi\right\rangle<\infty
$$

by the decay assumption on $v$.

On the other hand, if we assume that $\psi \in L^{2}$ with $H \psi=0$, we have that $c_{0}=0$, and hence by Lemma 5.3 we have $\phi=w \psi \in S_{2} L^{2}$. We need to prove that $S_{2} v G_{1} v S_{2} \phi=0$. Note that, as operators on $L^{2}, S_{2} v G_{1} v S_{2}=S_{2} v W v S_{2}$, where $W$ is the integral operator with kernel $-2 x \cdot y$. This is because $G_{1}(x, y)=|x-y|^{2}=$ $|x|^{2}-2 x \cdot y+|y|^{2}$, and the contribution of $|x|^{2}+|y|^{2}$ is zero since $P S_{2}=S_{2} P=0$.

We claim that if $\psi \in L^{2}$ with $H \psi=0$, then

$$
\int_{\mathbb{R}^{2}} y v(y) \phi(y) d y=0 .
$$

This implies that

$$
S_{2} v G_{1} v S_{2} \phi=S_{2} v W v S_{2} \phi=-2 S_{2} v(x) x \cdot \int_{\mathbb{R}^{2}} y v(y) \phi(y) d y=0,
$$

and hence $\phi \in S_{3} L^{2}$.

It remains to prove the claim above. In what follows below we can assume that $|x|>4$ since $\psi \in L^{\infty}$. Define the set $B:=\left\{y \in \mathbb{R}^{2}:|y|<|x| / 8\right\}$. Recall that we have $\psi=-G_{0} v \phi$, and as $P \phi=0$ we have

$$
\begin{aligned}
& \psi(x)=\frac{1}{4 \pi} \int_{\mathbb{R}^{2}} \ln \left(\frac{|x-y|^{2}}{|x|^{2}}\right) v(y) \phi(y) d y \\
& =\frac{1}{4 \pi} \int_{B} \ln \left(\frac{|x-y|^{2}}{|x|^{2}}\right) v(y) \phi(y) d y+\frac{1}{4 \pi} \int_{\mathbb{R}^{2} \backslash B} \ln \left(\frac{|x-y|^{2}}{|x|^{2}}\right) v(y) \phi(y) d y .
\end{aligned}
$$

First we note that the second term is in $L^{2}$. Indeed, using (77) and then $1 \lesssim\langle y\rangle /\langle x\rangle$, we see that

$$
\begin{aligned}
& \left|\int_{\mathbb{R}^{2} \backslash B} \ln \left(\frac{|x-y|^{2}}{|x|^{2}}\right) v(y) \phi(y) d y\right| \lesssim \int_{\mathbb{R}^{2} \backslash B}\left(1+|y|^{0+}+|x-y|^{0-}\right)|v(y) \phi(y)| d y \\
& \quad \lesssim \frac{1}{\langle x\rangle^{1+}} \int_{\mathbb{R}^{2} \backslash B}\langle y\rangle^{1+}\left(1+|y|^{0+}+|x-y|^{0-}\right)|v(y) \phi(y)| d y \lesssim \frac{1}{\langle x\rangle^{1+}} \in L^{2}\left(\mathbb{R}^{2}\right) .
\end{aligned}
$$


We now examine the integral on $B$. We note that on $B,\left.|| y\right|^{2}-\left.2 x \cdot y|/| x\right|^{2}<\frac{1}{2}$, and hence

$$
\ln \left(\frac{|x-y|^{2}}{|x|^{2}}\right)=\ln \left(1+\frac{|y|^{2}}{|x|^{2}}-\frac{2 x \cdot y}{|x|^{2}}\right)=-\frac{2 x \cdot y}{|x|^{2}}+O\left(\frac{\langle y\rangle^{1+}}{\langle x\rangle^{1+}}\right),
$$

so that

$$
\begin{aligned}
\frac{1}{4 \pi} \int_{B} \ln \left(\frac{|x-y|^{2}}{|x|^{2}}\right) v(y) \phi(y) d y= & -\frac{x}{2 \pi|x|^{2}} \cdot \int_{B} y v(y) \phi(y) d y \\
& +O\left(\frac{\int_{B}\langle y\rangle^{1+}|v(y) \phi(y)| d y}{\langle x\rangle^{1+}}\right) .
\end{aligned}
$$

The error term is in $L^{2}$. We also note that

$$
\left|\int_{\mathbb{R}^{2} \backslash B} \frac{x \cdot y}{|x|^{2}} v(y) \phi(y) d y\right| \lesssim \int_{\mathbb{R}^{2} \backslash B} \frac{\langle y\rangle^{1+}}{\langle x\rangle^{1+}}|v(y) \phi(y)| d y \lesssim\langle x\rangle^{-1-} \in L^{2}\left(\mathbb{R}^{2}\right) .
$$

Therefore, we can rewrite the main term as

$$
-\frac{x}{2 \pi|x|^{2}} \cdot \int_{B} y v(y) \phi(y) d y=-\frac{x}{2 \pi|x|^{2}} \cdot \int_{\mathbb{R}^{2}} y v(y) \phi(y) d y+O_{L^{2}}(1) .
$$

Using this in (82), we obtain

$$
\psi(x)=\Psi(x)-\frac{x}{2 \pi|x|^{2}} \cdot \int_{\mathbb{R}^{2}} y v(y) \phi(y) d y
$$

with $\Psi \in L^{2}$. As $x /|x|^{2}$ is not in $L^{2}\left(\mathbb{R}^{2}\right)$, we must have (81).

Lemma 5.6. The operator $G_{0} v S_{3}\left[S_{3} v G_{2} v S_{3}\right]^{-1} S_{3} v G_{0}$ is the orthogonal projection on $L^{2}$ onto the zero energy eigenspace of $H=-\Delta+V$.

Proof. Let $\left\{\phi_{j}\right\}_{j=1}^{N}$ be an orthonormal basis for the $S_{3} L^{2}$, the range of $S_{3}$. Then, we have

$$
\phi_{j}+w G_{0} v \phi_{j}=0, \quad 1 \leq j \leq N .
$$

We have $\phi_{j}=w \psi_{j}$ for each $j$ with $\psi_{j} \in L^{2}$. Since $P S_{3}=0$, we also have

$$
\int_{\mathbb{R}^{2}} V(x) \psi_{j}(x) d x=\int_{\mathbb{R}^{2}} v(x) \phi_{j}(x) d x=0 .
$$

Since $\left\{\phi_{j}\right\}_{j=1}^{N}$ is linearly independent, we have that $\left\{\psi_{j}\right\}_{j=1}^{N}$ is linearly independent, and it follows from (83) that

$$
\psi_{j}+G_{0} V \psi_{j}=0, \quad 1 \leq j \leq N .
$$

Using the orthonormal basis for $S_{3} L^{2}$, we have that for any $f \in L^{2}\left(\mathbb{R}^{2}\right), S_{3} f=$ $\sum_{j=1}^{N}\left\langle f, \phi_{j}\right\rangle \phi_{j}$. Therefore, we have

$$
S_{3} v G_{0} f=\sum_{j=1}^{N}\left\langle f, G_{0} v \phi_{j}\right\rangle \phi_{j}=-\sum_{j=1}^{N}\left\langle f, \psi_{j}\right\rangle \phi_{j}
$$


Let $A=\left\{A_{i j}\right\}_{i, j=1}^{N}$ be the matrix representation of $S_{3} v G_{2} v S_{3}$ with respect to the orthonormal basis of $S_{3} L^{2}$. Using (80),

$$
A_{i j}=\left\langle\phi_{i}, S_{3} v G_{2} v S_{3} \phi_{j}\right\rangle=\left\langle G_{0} v \phi_{i}, G_{0} v \phi_{j}\right\rangle=\left\langle G_{0} V \psi_{i}, G_{0} V \psi_{j}\right\rangle=\left\langle\psi_{i}, \psi_{j}\right\rangle .
$$

Let $P_{e}:=G_{0} v S_{3}\left[S_{3} v G_{2} v S_{3}\right]^{-1} S_{3} v G_{0}$. Then by (184), for any $f \in L^{2}\left(\mathbb{R}^{2}\right)$,

$$
\begin{aligned}
P_{e} f & =-\sum_{j=1}^{N} G_{0} v S_{3}\left[S_{3} v G_{2} v S_{3}\right]^{-1} \phi_{j}\left\langle f, \psi_{j}\right\rangle \\
& =-\sum_{i, j=1}^{N} G_{0} v S_{3}\left(A^{-1}\right)_{i j} \phi_{i}\left\langle f, \psi_{j}\right\rangle=\sum_{i, j=1}^{N} \psi_{i}\left(A^{-1}\right)_{i j}\left\langle f, \psi_{j}\right\rangle .
\end{aligned}
$$

Note that for $f=\psi_{k}, 1 \leq k \leq N$,

$$
P_{e} \psi_{k}=\sum_{i, j=1}^{N} \psi_{i}\left(A^{-1}\right)_{i j}\left\langle\psi_{k}, \psi_{j}\right\rangle=\sum_{i, j=1}^{N} \psi_{i}\left(A^{-1}\right)_{i j} A_{j k}=\psi_{k} .
$$

Thus, we can conclude that the range of $P_{e}$ is equal to the span of $\left\{\psi_{j}\right\}_{j=1}^{N}$ and that $P_{e}$ is the identity on the range of $P_{e}$. Since $P_{e}$ is self-adjoint, the claim is proven.

\section{A weighted estimate}

In this section we prove Theorem 1.2. Recall that if zero is an eigenvalue but there are neither s-wave nor p-wave resonances at zero, then $S_{1}=S_{2}=S_{3} \neq 0$. We note that in this case many terms in the expansions of $M^{ \pm}(\lambda)^{-1}$ in Corollaries 2.7 and 4.2 disappear. This follows, as now

$$
P S_{1}=S_{1} P=0, \quad S_{1} T P=P T S_{1}=0, \quad S_{1} v G_{1} v S_{1}=0 .
$$

We will also need a finer expansion for $M_{0}(\lambda)$ than is given in Lemma 2.2 to prove the theorem. Define $g_{2}^{ \pm}(\lambda)=\lambda^{4}\left(a_{2} \log \lambda+b_{2, \pm}\right)$ and $g_{3}(\lambda)=a_{3} \lambda^{4}$ with $a_{2}, a_{3} \in \mathbb{R} \backslash\{0\}$ and $b_{2,-}=\overline{b_{2,+}}$. Also let $G_{3}$ be the integral operator with the kernel $|x-y|^{4}$, and $G_{4}$ with the kernel $|x-y|^{4} \log |x-y|$. Similar to the expansion given in Lemma 2.2, we obtain

$$
M_{0}^{ \pm}(\lambda)=g_{1}^{ \pm}(\lambda) v G_{1} v+\lambda^{2} v G_{2} v+g_{2}^{ \pm}(\lambda) v G_{3} v+g_{3}(\lambda) v G_{4} v+\mathcal{O}_{1}\left(\lambda^{9 / 2}\right)
$$

by expanding the Bessel functions to order $z^{6} \log z$ and estimating the error term as in Lemma 2.2. This requires that $|V(x)| \lesssim\langle x\rangle^{-11-}$.

Proposition 6.1. Assume that $S_{1}=S_{2}=S_{3}$ and that $|V(x)| \lesssim\langle x\rangle^{-11-}$. Then, $B_{ \pm}$is invertible on $S_{1} L^{2}\left(\mathbb{R}^{2}\right)$, and we have

$$
\begin{array}{r}
B_{ \pm}^{-1}=\frac{D_{3}}{\lambda^{2}}+\frac{g_{2}^{ \pm}(\lambda)}{\lambda^{4}} D_{3} \Gamma_{2} D_{3}+D_{3} \Gamma_{3} D_{3}+\frac{g_{1}^{ \pm}(\lambda)^{2}}{\lambda^{4} h^{ \pm}(\lambda)} D_{3} \Gamma_{4} D_{3}+\frac{1}{h^{ \pm}(\lambda)} D_{3} \Gamma_{5} D_{3} \\
+\frac{g_{1}^{ \pm}(\lambda)}{\lambda^{2} h^{ \pm}(\lambda)} D_{3} \Gamma_{6} D_{3}+\mathcal{O}_{1}\left(\lambda^{1 / 2}\right)
\end{array}
$$

where $\Gamma_{i}$ are real-valued, absolutely bounded operators on $L^{2}$. 
Proof. We will modify the proof of Proposition 4.1. Using (85) in (62) we see that $B=E(\lambda)$, where (from (60) $)$

$$
\begin{aligned}
E(\lambda)= & S_{1} A^{-1}(\lambda) M_{0}(\lambda) A^{-1}(\lambda) S_{1} \\
& -S_{1} A^{-1}(\lambda)\left[M_{0}(\lambda) A^{-1}(\lambda)\right]^{2}\left[\mathbb{1}+M_{0}(\lambda) A^{-1}(\lambda)\right]^{-1} S_{1} .
\end{aligned}
$$

Since $S_{1}=S_{2}=S_{3}$, using (85) and (23) we have

$$
S_{1} A^{-1}(\lambda)=A^{-1}(\lambda) S_{1}=S_{3} .
$$

Using this, Lemma 2.2 and the fact that $A^{-1}(\lambda)=\mathcal{O}_{1}(1)$, we obtain

$$
B=E(\lambda)=S_{3} M_{0}(\lambda) S_{3}-S_{3} M_{0}(\lambda) A^{-1}(\lambda) M_{0}(\lambda) S_{3}+\mathcal{O}_{1}\left(\lambda^{9 / 2}\right) .
$$

Using (86) and the fact that $S_{3} v G_{1} v S_{3}=0$, we get

$$
S_{3} M_{0}(\lambda) S_{3}=\lambda^{2} S_{3} v G_{2} v S_{3}+g_{2}(\lambda) S_{3} v G_{3} v S_{3}+g_{3}(\lambda) S_{3} v G_{4} v S_{3}+\mathcal{O}_{1}\left(\lambda^{9 / 2}\right) .
$$

We now note that by writing $G_{1}(x, y)=|x|^{2}-2 x \cdot y+|y|^{2}$ and using (81) and $P \perp Q>S_{3}$, one obtains

$$
S_{3} v G_{1} v Q=Q v G_{1} v S_{3}=0 .
$$

Using this and (85) in (23) and (86), we have

$$
\begin{aligned}
S_{3} M_{0}(\lambda) A^{-1}(\lambda) M_{0}(\lambda) S_{3} & =\frac{g_{1}(\lambda)^{2}}{h(\lambda)} S_{3} v G_{1} v P v G_{1} v S_{3}+\frac{\lambda^{4}}{h(\lambda)} S_{3} v G_{2} v S v G_{2} v S_{3} \\
& +\frac{\lambda^{2} g_{1}(\lambda)}{h(\lambda)}\left[S_{3} v G_{1} v S v G_{2} v S_{3}+S_{3} v G_{2} v S v G_{1} v S_{3}\right] \\
& +\lambda^{4} S_{3} v G_{2} v Q D_{0} Q v G_{2} v S_{3}+\mathcal{O}_{1}\left(\lambda^{6-}\right) .
\end{aligned}
$$

Therefore, using these expansions in (89), we have

$$
B=\lambda^{2} \Gamma_{1}+g_{2}^{ \pm}(\lambda) \Gamma_{2}+\lambda^{4} \Gamma_{3}+\frac{g_{1}^{ \pm}(\lambda)^{2}}{h^{ \pm}(\lambda)} \Gamma_{4}+\frac{\lambda^{4}}{h^{ \pm}(\lambda)} \Gamma_{5}+\frac{\lambda^{2} g_{1}^{ \pm}(\lambda)}{h^{ \pm}(\lambda)} \Gamma_{6}+\mathcal{O}_{1}\left(\lambda^{9 / 2}\right),
$$

where $\Gamma_{i}$ are absolutely bounded operators on $L^{2}$ with $\Gamma_{i}=S_{3} \Gamma_{i} S_{3}$, and $\Gamma_{1}^{-1}=D_{3}$. Inverting this via Neumann series yields the claim of the proposition.

Corollary 6.2. Assume that $S_{1}=S_{2}=S_{3}$ and that $|V(x)| \lesssim\langle x\rangle^{-11-}$. Then

$$
\begin{aligned}
M^{ \pm}(\lambda)^{-1}=\frac{D_{3}}{\lambda^{2}}+\left(a_{1} \log \lambda+\right. & \left.b_{1, \pm}\right) \Xi_{1}+\left(1+\frac{b_{3, \pm}}{a_{2} \log \lambda+b_{2, \pm}}\right) \Xi_{2} \\
& +\frac{1}{h^{ \pm}(\lambda)} \Xi_{3}+\left(M^{ \pm}(\lambda)+S_{1}\right)^{-1}+\mathcal{O}_{1}\left(\lambda^{1 / 2}\right) .
\end{aligned}
$$

Here, $\Xi_{i}$ are real-valued absolutely bounded operators, $\Xi_{2}$ and $\Xi_{3}$ have a projection orthogonal to $P$ on at least one side, and $\Xi_{1}$ have orthogonal projections on both sides. Further, $a_{i} \in \mathbb{R} \backslash\{0\}$ and $b_{i,+}=\overline{b_{i,-}}$.

We should note that in the statement of the corollary we listed only one term of each form. For example, there are several different terms of the form $\frac{b_{3, \pm}}{a_{2} \log \lambda+b_{2, \pm}} \Xi_{2}$ in the expansion. 
Proof of Corollary 6.2. Using (24), (23) and (88), and then (85) and (90), we have

$$
\begin{aligned}
\left(M(\lambda)+S_{1}\right)^{-1} S_{3}= & S_{3}+A^{-1}(\lambda) M_{0}(\lambda) S_{3}+\mathcal{O}_{1}\left(\lambda^{4-}\right) \\
= & S_{3}+\frac{g_{1}(\lambda)}{h(\lambda)}\left[P v G_{1} v S_{3}-Q D_{0} Q T P v G_{1} v S_{3}\right] \\
& +\lambda^{2} Q D_{0} Q v G_{2} v S_{3}+\frac{\lambda^{2}}{h(\lambda)} S v G_{2} v S_{3}+\mathcal{O}_{1}\left(\lambda^{4-}\right), \\
S_{3}\left(M(\lambda)+S_{1}\right)^{-1}= & S_{3}+S_{3} M_{0}(\lambda) A^{-1}(\lambda)+\mathcal{O}_{1}\left(\lambda^{4-}\right) \\
= & S_{3}+\frac{g_{1}(\lambda)}{h(\lambda)}\left[S_{3} v G_{1} v P-S_{3} v G_{1} v P T Q D_{0} Q\right] \\
& +\lambda^{2} S_{3} v G_{2} v Q D_{0} Q+\frac{\lambda^{2}}{h(\lambda)} S_{3} v G_{2} v S+\mathcal{O}_{1}\left(\lambda^{4-}\right) .
\end{aligned}
$$

Using these and (87) yields that

$$
\begin{aligned}
\left(M(\lambda)+S_{1}\right)^{-1} S_{3} B^{-1} S_{3}( & \left.M(\lambda)+S_{1}\right)^{-1}=\frac{D_{3}}{\lambda^{2}}+\left(a_{1} \log \lambda+b_{1, \pm}\right) \Xi_{1} \\
& +\left(1+\frac{b_{3, \pm}}{a_{2} \log \lambda+b_{2, \pm}}\right) \Xi_{2}+\frac{1}{h^{ \pm}(\lambda)} \Xi_{3}+\mathcal{O}_{1}\left(\lambda^{1 / 2}\right) .
\end{aligned}
$$

Applying Lemma 2.4 finishes the proof.

Using Corollary 6.2 and Lemma [5.6 in (27), we see that the contribution of the $D_{3} / \lambda^{2}$ term can be written as

$$
\begin{aligned}
& R_{0}^{+}\left(\lambda^{2}\right) v \frac{D_{3}}{\lambda^{2}} v R_{0}^{+}\left(\lambda^{2}\right)=\frac{1}{\lambda^{2}}\left(R_{0}^{+}\left(\lambda^{2}\right)-g^{+}(\lambda)\right) v D_{3} v\left(R_{0}^{+}\left(\lambda^{2}\right)-g^{+}(\lambda)\right) \\
&(92) \quad=\frac{1}{\lambda^{2}}\left(R_{0}^{+}\left(\lambda^{2}\right)-g^{+}(\lambda)-G_{0}\right) v D_{3} v\left(R_{0}^{+}\left(\lambda^{2}\right)-g^{+}(\lambda)-G_{0}\right) \\
&(93) \quad+\frac{1}{\lambda^{2}} G_{0} v D_{3} v\left(R_{0}^{+}\left(\lambda^{2}\right)-g^{+}(\lambda)-G_{0}\right)+\frac{1}{\lambda^{2}}\left(R_{0}^{+}\left(\lambda^{2}\right)-g^{+}(\lambda)-G_{0}\right) v D_{3} v G_{0} \\
&(94) \quad+\frac{1}{\lambda^{2}} P_{e} .
\end{aligned}
$$

In the first line above, we used the fact that $P D_{3}=D_{3} P=0$ to subtract off $g^{+}(\lambda)$.

Lemma 6.3. Under the assumptions of Theorem 1.2, if we project away from the eigenspace of $H=-\Delta+V$ at zero energy, for Schwartz functions $f$ and $g$ the following bound holds:

$$
\begin{aligned}
& \left|\int_{0}^{\infty} \frac{e^{i t \lambda^{2}} \chi(\lambda)}{\lambda}\left\langle\left[R_{0}^{+}\left(\lambda^{2}\right) v D_{3} v R_{0}^{+}\left(\lambda^{2}\right)-R_{0}^{-}\left(\lambda^{2}\right) v D_{3} v R_{0}^{-}\left(\lambda^{2}\right)\right] f, g\right\rangle d \lambda\right| \\
& \quad \lesssim|t|^{-1}\|f\|_{L^{1,1+}}\|g\|_{L^{1,1+}} .
\end{aligned}
$$

Proof. First note that since we project away from the zero eigenspace, the contribution of (94) cancels out. To bound the contribution of other terms, recall that the expansion for $R_{0}^{ \pm}\left(\lambda^{2}\right)$ used in Lemma 2.2 gives

$$
\begin{aligned}
\left|R_{0}^{+}\left(\lambda^{2}\right)(x, y)-g^{+}(\lambda)-G_{0}(x, y)\right| & \lesssim \lambda^{1+}|x-y|^{1+} \lesssim \lambda^{1+}\left(\langle x\rangle^{1+}+\langle y\rangle^{1+}\right), \\
\left|\frac{\partial}{\partial \lambda}\left(R_{0}^{+}\left(\lambda^{2}\right)(x, y)-g^{+}(\lambda)-G_{0}(x, y)\right)\right| & \lesssim \lambda^{0+}\left(\langle x\rangle^{1+}+\langle y\rangle^{1+}\right) .
\end{aligned}
$$


Note that if $|\Phi(\lambda)|+\lambda\left|\Phi^{\prime}(\lambda)\right| \lesssim \lambda^{1+}$ and the same for $\Psi$, then

$$
\left|\int_{0}^{\infty} \frac{e^{i t \lambda^{2}} \chi(\lambda)}{\lambda} \Phi(\lambda) \Psi(\lambda) d \lambda\right| \lesssim|t|^{-1} \int_{0}^{\infty}\left|\frac{d}{d \lambda}\left(\frac{\chi(\lambda) \Phi(\lambda) \Psi(\lambda)}{\lambda^{2}}\right)\right| d \lambda \lesssim|t|^{-1}
$$

Also using the fact that $\left|D_{3}\right|: L^{2} \rightarrow L^{2}$ and $\langle x\rangle^{1+} v(x) \in L^{2}$, the contribution of (92) satisfies the claim of the lemma.

For the contribution of the terms in (93), we need to use the cancellation between the '+' and '-' terms in Stone's formula:

$$
\begin{aligned}
G_{0} v D_{3} v\left(\left[R_{0}^{+}\left(\lambda^{2}\right)-g^{+}(\lambda)\right]-\left[R_{0}^{-}\left(\lambda^{2}\right)-g^{-}(\lambda)\right]\right) \\
=G_{0} v D_{3} v\left(2 i J_{0}(\lambda|\cdot|)-2 i \Im(z)\right)=2 i G_{0} v D_{3} v J_{0}(\lambda|\cdot|),
\end{aligned}
$$

where we used (33) in the last step. As in the case of an s-wave resonance, we separate into the high and low energies. For the low energy part we use (33) and investigate

$$
\int_{0}^{\infty} e^{i t \lambda^{2}} \frac{\chi(\lambda)}{\lambda}\left(\chi\left(\lambda\left|y-y_{1}\right|\right) J_{0}\left(\lambda\left|y-y_{1}\right|\right)-\chi\left(\lambda\left(1+\left|y_{1}\right|\right)\right) J_{0}\left(\lambda\left(1+\left|y_{1}\right|\right)\right)\right) d \lambda .
$$

After an integration by parts, the result relies on proving the following bound:

$$
\int_{0}^{1}\left|\frac{d}{d \lambda}\left(\frac{\chi\left(\lambda\left|y-y_{1}\right|\right) J_{0}\left(\lambda\left|y-y_{1}\right|\right)-\chi\left(\lambda\left(1+\left|y_{1}\right|\right) J_{0}\left(\lambda\left(1+\left|y_{1}\right|\right)\right)\right.}{\lambda^{2}}\right)\right| d \lambda<C_{y, y_{1}} .
$$

We need not consider when the derivative acts on the cut-off function, as this restricts us to an annulus where $\lambda \sim\left|y-y_{1}\right|^{-1}$ or $\lambda \sim\left(1+\left|y_{1}\right|\right)^{-1}$ and we can bound (96) by

$$
\int_{\lambda \sim\left|y-y_{1}\right|^{-1}} \frac{1}{\lambda^{2}} d \lambda \lesssim\left|y-y_{1}\right| \lesssim\langle y\rangle\left\langle y_{1}\right\rangle .
$$

The analogous bound holds for $\lambda \sim\left(1+\left|y_{1}\right|\right)^{-1}$. With this in mind, it suffices to prove that

$$
\left|\int_{0}^{1} \frac{f\left(\lambda\left|y-y_{1}\right|\right)-f\left(\lambda\left(1+\left|y_{1}\right|\right)\right)}{\lambda^{3}} d \lambda\right| \lesssim\langle y\rangle^{2+}\left\langle y_{1}\right\rangle^{1+}
$$

with $f(z):=\chi(z)\left[-2 J_{0}(z)+z J_{0}^{\prime}(z)\right]$. As we are restricted to low energy, we have the expansion for $J_{0}(z)$ and its derivative in a power of $z$ from (5) so that

$$
f(z)=a_{0}+a_{1} z+a_{2} z^{2}+O\left(z^{2+}\right) .
$$

Since $f(0)=-2 J_{0}(0)=-2$ we have that $a_{0}=-2$. Further, $f^{\prime}(0)=-J_{0}^{\prime}(0)+$ $\left.z J_{0}^{\prime \prime}(z)\right|_{z=0}=0$ and $f^{\prime \prime}(z)=z J_{0}^{\prime \prime \prime}(z)$. Therefore, we have $a_{1}=a_{2}=0$ and

$$
f(z)=-2+O\left(z^{2+}\right), \quad f^{\prime}(z)=O\left(z^{1+}\right) .
$$

Now,

$$
\begin{aligned}
& \left|f\left(\lambda\left|y-y_{1}\right|\right)-f\left(\lambda\left(1+\left|y_{1}\right|\right)\right)\right|=\left|\int_{\lambda\left(1+\left|y_{1}\right|\right)}^{\lambda\left|y-y_{1}\right|} f^{\prime}(z) d z\right| \\
& \lesssim \lambda^{2+}|| y-y_{1}\left|-\left(1+\left|y_{1}\right|\right)\right|\left(\left|y-y_{1}\right|^{1+}+\left\langle y_{1}\right\rangle^{1+}\right) \lesssim \lambda^{2+}\langle y\rangle^{2+}\left\langle y_{1}\right\rangle^{1+} .
\end{aligned}
$$


Thus, we have

$$
\text { (196) } \begin{aligned}
& \lesssim\left|\int_{0}^{1} \frac{f\left(\lambda\left|y-y_{1}\right|\right)-f\left(\lambda\left(1+\left|y_{1}\right|\right)\right)}{\lambda^{3}} d \lambda\right| \\
& \lesssim\langle y\rangle^{2+}\left\langle y_{1}\right\rangle^{1+} \int_{0}^{1} \lambda^{-1+} d \lambda \lesssim\langle y\rangle^{2+}\left\langle y_{1}\right\rangle^{1+},
\end{aligned}
$$

as desired.

Now, for the high energy we proceed along the lines of the proof of Lemma 3.8. We employ the function $\widetilde{G}^{ \pm}\left(\lambda,\left|y-y_{1}\right|, 1+\left|y_{1}\right|\right)$ of Lemma 3.7. Specifically, we need to bound

$$
\int_{0}^{\infty} e^{i t \lambda^{2}} \chi(\lambda) G_{0} \frac{v D_{3} v}{\lambda} \widetilde{G}^{ \pm}(\lambda, p, q) d \lambda
$$

with $p=\max \left(\left|y-y_{1}\right|, 1+\left|y_{1}\right|\right)$ and $q=\min \left(\left|y-y_{1}\right|, 1+\left|y_{1}\right|\right)$. We will apply Lemma 3.6 to

$$
a(\lambda)=\frac{\chi(\lambda) \widetilde{G}^{ \pm}(\lambda)}{\lambda} .
$$

Using the bounds of Lemma 3.7 with $\tau=1$, we have

$$
\begin{aligned}
& |a(\lambda)| \lesssim\langle y\rangle\left(\frac{\tilde{\chi}(\lambda p)}{|\lambda p|^{1 / 2}}+\frac{\tilde{\chi}(\lambda q)}{|\lambda q|^{1 / 2}}\right) \lesssim\langle y\rangle(\widetilde{\chi}(\lambda p) \sqrt{p \lambda}+\widetilde{\chi}(\lambda q) \sqrt{q \lambda}), \\
& \left|a^{\prime}(\lambda)\right| \lesssim \frac{1}{\lambda}\langle y\rangle\left(\frac{\tilde{\chi}(\lambda p)}{|\lambda p|^{1 / 2}}+\frac{\tilde{\chi}(\lambda q)}{|\lambda q|^{1 / 2}}\right) \lesssim\langle y\rangle\left(\frac{p \widetilde{\chi}(\lambda p)}{|\lambda p|^{1 / 2}}+\frac{q \widetilde{\chi}(\lambda q)}{|\lambda q|^{1 / 2}}\right) .
\end{aligned}
$$

Here we used the fact that on the support of $\widetilde{\chi}(\lambda p)$, we have $1 \lesssim \lambda p$. At this point, the proof follows exactly along the lines of Lemma 3.8 with the extra weights of $p+q \lesssim\langle y\rangle\left\langle y_{1}\right\rangle$, which yields the required bound.

We are now ready to prove the theorem. We provide a sketch, as there is a significant overlap with the proofs of previous estimates in Section 3

Proof of Theorem 1.2. We already proved the theorem for the contribution of the $D_{3} / \lambda^{2}$ term in Corollary 6.2 to (27). The contribution of the $\Xi_{1}$ term and the terms in the second line of (91) satisfies the dispersive bound by the results of Section 3 . It remains to control the contribution of

$$
\left(1+\frac{b_{3, \pm}}{a_{2} \log \lambda+b_{2, \pm}}\right) \Xi_{2}
$$

We will only provide a brief sketch. Recall that $\Xi_{2}$ has projection orthogonal to $P$ only on one side, say on the right. On high energy, we can use $\lambda\left|x-x_{1}\right| \gtrsim 1$ to extract positive powers of $\lambda$ for the integration at the loss of a weight as in the proof of Lemma 6.3. The polynomial weights arising are either ameliorated by the decay of the potential $v$ or go into the weight of the weighted dispersive bound. For the low energy part, the worst case is when we have $Y_{0}$ on both sides. This arises only with the term containing $\log \lambda$ in the denominator, due to the cancellation between the \pm terms. On the right hand side, using (33), we replace $\chi Y_{0}$ with $F$ from Lemma 3.3 to reduce to bounding the following integral:

$$
\left|\int_{0}^{\infty} e^{i t \lambda^{2}} \lambda \chi(\lambda) \chi\left(\lambda\left|x-x_{1}\right|\right) Y_{0}\left(\lambda\left|x-x_{1}\right|\right) \frac{b_{3}}{a_{2} \log \lambda+b_{2}} F\left(\lambda, y, y_{1}\right) d \lambda\right| .
$$


After an integration by parts, it suffices to prove that

$$
\begin{aligned}
\int_{0}^{\infty} \mid \frac{d}{d \lambda}\left\{\chi(\lambda) \chi\left(\lambda\left|x-x_{1}\right|\right) Y_{0}(\lambda \mid x\right. & \left.\left.-x_{1} \mid\right) \frac{b_{3}}{a_{2} \log \lambda+b_{2}} F\left(\lambda, y, y_{1}\right)\right\} \mid d \lambda \\
& \lesssim k\left(x, x_{1}\right) k\left(y, y_{1}\right)\langle x\rangle^{1+}\langle y\rangle^{1+}\left\langle x_{1}\right\rangle^{1+}\left\langle y_{1}\right\rangle^{1+} .
\end{aligned}
$$

We note, from (6), that

$$
\chi\left(\lambda\left|x-x_{1}\right|\right) Y_{0}\left(\lambda\left|x-x_{1}\right|\right)=\frac{2}{\pi}\left[\log \lambda+\log \left|x-x_{1}\right|+\gamma\right]+O\left(\lambda^{1+}\left|x-x_{1}\right|^{1+}\right) .
$$

The first $\log \lambda$ is the most troubling. We note that to control it we use the following facts:

$$
\left|\log (\lambda) \frac{b_{3}}{a_{2} \log \lambda+b_{2}}\right| \lesssim 1, \quad\left|\frac{d}{d \lambda}\left(\log (\lambda) \frac{b_{3}}{a_{2} \log \lambda+b_{2}}\right)\right| \lesssim \frac{1}{\lambda(\log \lambda)^{2}} .
$$

The contribution of the other terms can be bounded by similar arguments.

\section{REFERENCES}

[1] Abramowitz, M. and I. A. Stegun. Handbook of mathematical functions with formulas, graphs, and mathematical tables. National Bureau of Standards Applied Mathematics Series, 55. For sale by the Superintendent of Documents, U.S. Government Printing Office, Washington, D.C. 1964 MR0167642 (29:4914)

[2] Agmon, S. Spectral properties of Schrödinger operators and scattering theory. Ann. Scuola Norm. Sup. Pisa Cl. Sci. (4) 2 (1975), no. 2, 151-218. MR0397194 (53:1053)

[3] Bollé, D., Danneels, C., and F. Gesztesy. Threshold scattering in two dimensions. Ann. Inst. H. Poincaré Phys. Théor. 48 (1988), no. 2, 175-204. MR952661 (89k:81184)

[4] Bollé, D., Gesztesy, F., and S. F. Wilk. A complete treatment of low-energy scattering in one dimension. J. Operator Theory 13 (1985), no. 1, 3-31. MR768299 (86f:34047)

[5] Erdoğan, M. B., and W.R. Green. A weighted dispersive estimate for Schrödinger operators in dimension two. Preprint (2012): Accepted by Comm. Math. Phys.

[6] Erdoğan, M. B., and W. Schlag. Dispersive estimates for Schrödinger operators in the presence of a resonance and/or an eigenvalue at zero energy in dimension three: I. Dynamics of PDE 1 (2004), 359-379. MR2127577 (2006j:35036)

[7] Erdoğan, M. B., and W. Schlag. Dispersive estimates for Schrödinger operators in the presence of a resonance and/or eigenvalue at zero energy in dimension three: II. J. Anal. Math. 99 (2006), 199-248. MR2279551 (2008e:35034)

[8] Goldberg, M. A Dispersive Bound for Three-dimensional Schrödinger operators with zero energy eigenvalues. Comm. PDE 35 (2010), 1610-1634. MR2754057

[9] Goldberg M., Dispersive bounds for the three-dimensional Schrödinger equation with almost critical potentials. Geom. and Funct. Anal. vol. 16, no. 3 (2006), 517-536. MR2238943 (2007g:35026)

[10] Goldberg, M. Dispersive Estimates for the Three-Dimensional Schrödinger Equation with Rough Potentials. Amer. J. Math. 128 (2006) 731-750. MR2230923 (2007g:35025)

[11] Goldberg, M., and W. Schlag. Dispersive estimates for Schrödinger operators in dimensions one and three. Comm. Math. Phys. vol. 251, no. 1 (2004), 157-178. MR2096737(2005g:81339)

[12] Jensen, A. Spectral properties of Schrödinger operators and time-decay of the wave functions results in $L^{2}\left(R^{m}\right), m \geq 5$. Duke Math. J. 47 (1980), no. 1, 57-80. MR.563367 (81e:35092)

[13] Jensen, A. Spectral properties of Schrödinger operators and time-decay of the wave functions. Results in $L^{2}\left(R^{4}\right)$. J. Math. Anal. Appl. 101 (1984), no. 2, 397-422. MR748579 (85j:35138)

[14] Jensen, A., and T. Kato. Spectral properties of Schrödinger operators and time-decay of the wave functions. Duke Math. J. 46 (1979), no. 3, 583-611. MR544248 (81b:35079)

[15] Jensen, A., and G. Nenciu. A unified approach to resolvent expansions at thresholds. Rev. Mat. Phys. vol. 13, no. 6 (2001), 717-754. MR1841744 (2002e:81031)

[16] Jensen, A., and K. Yajima. A remark on $L^{p}$-boundedness of wave operators for twodimensional Schrödinger operators. Comm. Math. Phys. 225 (2002), no. 3, 633-637. MR.1888876 (2002k:81276) 
[17] Journé, J.-L., Soffer, A., and C. D. Sogge. Decay estimates for Schrödinger operators. Comm. Pure Appl. Math. 44 (1991), no. 5, 573-604. MR.1105875 (93d:35034)

[18] Moulin, S. High frequency dispersive estimates in dimension two. Ann. Henri Poincaré 10 (2009), no. 2, 415-428. MR2511893 (2010j:35094)

[19] Murata, M. Asymptotic expansions in time for solutions of Schrödinger-type equations. J. Funct. Anal. 49 (1) (1982), 10-56. MR680855 (85d:35019)

[20] Rauch, J. Local decay of scattering solutions to Schrödinger's equation. Comm. Math. Phys. 61 (1978), no. 2, 149-168. MR0495958 (58:14590)

[21] Reed, M., and B. Simon. Methods of Modern Mathematical Physics I: Functional Analysis, IV: Analysis of Operators, Academic Press, New York, NY, 1972. MR0493419 (58:12429a)

[22] Rodnianski, I., and W. Schlag. Time decay for solutions of Schrödinger equations with rough and time-dependent potentials. Invent. Math. 155 (2004), no. 3, 451-513. MR2038194 (2005h:35295)

[23] Schlag, W. Dispersive estimates for Schrödinger operators: A survey. Mathematical aspects of nonlinear dispersive equations, 255-285, Ann. of Math. Stud., 163, Princeton Univ. Press, Princeton, NJ, 2007. MR2333215 (2009k:35043)

[24] Schlag, W. Dispersive estimates for Schrödinger operators in dimension two. Comm. Math. Physi. 257 (2005), no. 1, 87-117. MR2163570(2006d:35045)

[25] Stoiciu, M. An estimate for the number of bound states of the Schrödinger operator in two dimensions. Proc. Amer. Math. Soc. 132 (2004), no. 4, 1143-1151. MR2045431(2005c:81061)

[26] Weder, R. $L^{p}-L^{p^{\prime}}$ estimates for the Schrödinger equation on the line and inverse scattering for the nonlinear Schrödinger equation with a potential. J. Funct. Anal. 170 (2000), no. 1, 37-68. MR1736195(2001e:35135)

[27] Yajima, K. L ${ }^{p}$-boundedness of wave operators for two-dimensional Schrödinger operators. Comm. Math. Phys. 208 (1999), no. 1, 125-152. MR.1729881(2001i:35232)

[28] Yajima, K. Dispersive estimate for Schrödinger equations with threshold resonance and eigenvalue, Comm. Math. Phys. 259 (2005), 475-509. MR2172692 (2006h:35042)

Department of Mathematics, University of Illinois, Urbana, Illinois 61801

E-mail address: berdogan@math.uiuc.edu

Department of Mathematics and Computer Science, Eastern Illinois University, Charleston, Illinois 61920

E-mail address: wrgreen2@eiu.edu

Current address: Department of Mathematics, Rose-Hulman Institute of Technology, Terre

Haute, Indiana 47803

E-mail address: green@rose-hulman.edu 